OPEN ACCESS

Edited by:

Sergio Davinelli,

University of Molise, Italy

Reviewed by:

Graziamaria Corbi,

University of Molise, Italy

Julien Bensalem,

South Australian Health and Medical

Research Institute (SAHMRI), Australia

${ }^{*}$ Correspondence:

A. Veronica Witte

witte@cbs.mpg.de

tThese authors have contributed equally to this work

Specialty section:

This article was submitted to

Clinical Nutrition,

a section of the journal

Frontiers in Nutrition

Received: 04 June 2021 Accepted: 20 December 2021

Published: 26 January 2022

Citation:

de Vries K, Medawar E, Korosi A and Witte AV (2022) The Effect of

Polyphenols on Working and Episodic

Memory in Non-pathological and

Pathological Aging: A Systematic

Review and Meta-Analysis.

Front. Nutr. 8:720756.

doi: 10.3389/fnut.2021.720756

\section{The Effect of Polyphenols on Working and Episodic Memory in Non-pathological and Pathological Aging: A Systematic Review and Meta-Analysis}

\author{
Karin de Vries ${ }^{1,2+}$, Evelyn Medawar ${ }^{2,3+}$, Aniko Korosi ${ }^{1}$ and A. Veronica Witte ${ }^{2,4 *}$ \\ ${ }^{1}$ Swammerdam Institute for Life Sciences, University of Amsterdam, Amsterdam, Netherlands, ${ }^{2}$ Department of Neurology, \\ Max Planck Institute for Human Cognitive and Brain Sciences, Leipzig, Germany, ${ }^{3}$ Faculty of Philosophy, Berlin School of \\ Mind and Brain, Humboldt-Universität zu Berlin, Berlin, Germany, ${ }^{4}$ Clinic for Cognitive Neurology, University Medical Center \\ Leipzig, Leipzig, Germany
}

Life expectancy steadily increases, and so do age-associated diseases, leading to a growing population suffering from cognitive decline and dementia. Impairments in working memory (WM) and episodic memory (EM) are associated with an increased risk of developing dementia. While there are no effective pharmacological therapies to preserve or enhance cognition and to slow down the progression from mild memory complaints to dementia so far, plant-based nutrients including polyphenols have been suggested to exert beneficial effects on brain aging. This review studies whether supplementary polyphenols are effective in preserving or enhancing memory in both non-pathological and pathological aging, and whether there are polyphenol efficiency differences between WM and EM. A systematic literature search was conducted and 66 out of 294 randomized clinical trials with 20 participants or more per group, aged 40 years or older were included. These covered a daily intake of 35-1,600 mg polyphenols, e.g., flavonols, flavonoids, isoflovones, anthocyanins, and/or stilbenes, over the course of 2 weeks to 6.5 years duration. In total, around half of the studies reported a significantly improved performance after polyphenol administration compared to control, while three studies reported a worsening of performance, and the remainder did not observe any effects. According to pooled WM and EM meta-analysis of all memory outcomes reported in 49 studies, overall effect size for WM and EM indicated a significant small positive effect on EM and WM with similar estimates $(b \sim 0.24, p<0.001)$, with large study heterogeneity and significant Funnel asymmetry tests suggesting a positivity bias. These results remained similar when excluding studies reporting extremely large positive effect sizes from the meta-analyses. While Ginkgo biloba and isoflavones did not show benefits in subgroup meta-analyses, those suggested some effects in extracts containing anthocyanins, other flavonoids and resveratrol, again potentially resulting from publication bias. To conclude, a systematic review and meta-analysis indicate that shortto moderate-term polyphenol interventions might improve WM and EM in middle-to older aged adults, however, publication bias in favor of positive results seems likely, 
rendering definite conclusions difficult. Future studies with larger, more diverse samples and sensitive monitoring of cardiovascular, metabolic and beginning brain pathologies as well as longer follow-up are needed to better understand the impact of age, (beginning) pathologies, gender, and long-term use on polyphenol action.

Keywords: polyphenol, RCT-randomized controlled trial, aging, episodic memory, working memory

\section{INTRODUCTION}

Due to economic, social, and health care developments, the life expectancy of people in all regions of the world is increasing. As a consequence, the proportion of people aged 65 or older is expected to rise from 1 in 11 people in 2019 to 1 in 6 people in 2050 (1). Aging is associated with deteriorating health, including brain health. With aging, for example, pro-inflammatory activity and less efficient anti-oxidative mechanisms lead to higher burden of neuroinflammation and oxidative damage in the brain (2). Moreover, an increase in neurodegeneration (i.e., loss of neurons) and a reduction in neurogenesis (i.e., formation of new neurons) occur with aging and negatively affect the neuronal plasticity of the brain $(3,4)$. These brain alterations are thought to underly cognitive decline and memory impairment, a key symptom of dementia such as in Alzheimer's disease (4). As the number of people with dementia could likely expand to 132 million people by 2050, causing extreme social and individual costs (5-7), healthy brain aging constitutes a global challenge. However, currently, there are no effective pharmacological therapies to preserve or enhance cognition in older age $(8,9)$.

While unhealthy lifestyle can accelerate the process of cognitive decline during aging, health-promoting lifestyle factors such as physical activity and nutrition might slow down the trajectory of cognitive decline (10). Therefore, the interest in studying the influence of polyphenols on cognitive functioning is rising. Polyphenols are micronutrients that are found in plantbased foods (4). There are several subclasses of polyphenols, of which flavonoids, stilbenes, and phenolic acids are examples (11). Multiple phenol groups per molecule characterize polyphenols, but the chemical properties of different polyphenols are heterogeneous (12). Studies suggest that polyphenols can cross the blood-brain barrier and affect aging processes due to their anti-inflammatory, antioxidant, and neuroprotective properties $(2,13)$. In the past $\sim 15$ years, numerous animal experiments and human studies have investigated whether polyphenols enhance cognitive performance or prevent age-related brain pathologies, yet the level of scientific evidence and clinical efficiency in humans still remain unclear [reviewed e.g., in (14-16)].

Therefore, we aimed to perform a systematic review whether polyphenols are effective in preserving or enhancing memory in (non-)pathological human aging. We decided to consider results from randomized-controlled trials (RCT) only, as this study design is the most ideal to demonstrate a causal relationship between an intervention and an effect and is an important step toward evidence-based therapies (17). We focused on the memory system because (1) memory decline may underlie changes in other cognitive functions and (2) memory complaints are reported first in the preclinical trajectory of cognitive decline, sometimes already 16 years before diagnosis (18, 19). Two common types of dementia, namely frontotemporal dementia and Alzheimer's disease, are characterized by earlystage deficits in working memory (WM) and episodic memory (EM) respectively, underlining (partly) independent neuronal underpinnings of the two memory processes (20). WM is required to direct attention and manipulate information that is stored in short-term memory $(21,22)$ and EM allows to learn, store, and retrieve information about personal experiences $(22,23)$. In addition, impairments in either WM and EM are associated with an increased risk of developing dementia $(24,25)$. We therefore studied both WM and EM and asked whether polyphenols are effective in preserving or enhancing memory in (non-)pathological human aging.

\section{METHODS}

\section{Literature Search}

A literature search was conducted in PubMed in August 2021 (Figure 1). We decided to The search term $((($ cognitive AND blueberry AND (humans[Filter])) OR (cognitive AND gingko AND (humans[Filter]))) OR (cognitive AND curcumin AND (humans[Filter]))) OR (cognitive AND polyphenol AND (humans[Filter]))) OR (cognitive AND flavonoids AND (humans[Filter])) together with the filter "clinical trial" or "clinical study" or "RCT" resulted in 294 hits. All articles were screened on the following inclusion criteria: (1) RCT study design, (2) administration of polyphenols or polyphenol-rich extracts or supplements, (3) an included measure of working or episodic memory, (4) a sample size of at least 20 participants per group with available follow-up data (i.e., completers), and (5) the participants included in the sample had to be at least 40 years old. Exclusion criteria were non-English articles, single dose trials, severe disease of participants (such as depression, multiple sclerosis, cancer) as well as duplicates or re-analyses of previously published trials. A study outcome measures was identified as memory performance-related and grouped into EM and WM, respectively, based on the author's descriptions and/or by inspecting the description of the used tests in the literature. No self-reported memory measures were evaluated. Note that several neuropsychological tests used in dementia patients, such as the ADAS-cog, usually do not provide raw memory subscale values. These studies were included in the systematic review but excluded from meta-analyses due to the lack of specificity regarding memory functions. A cutoff 


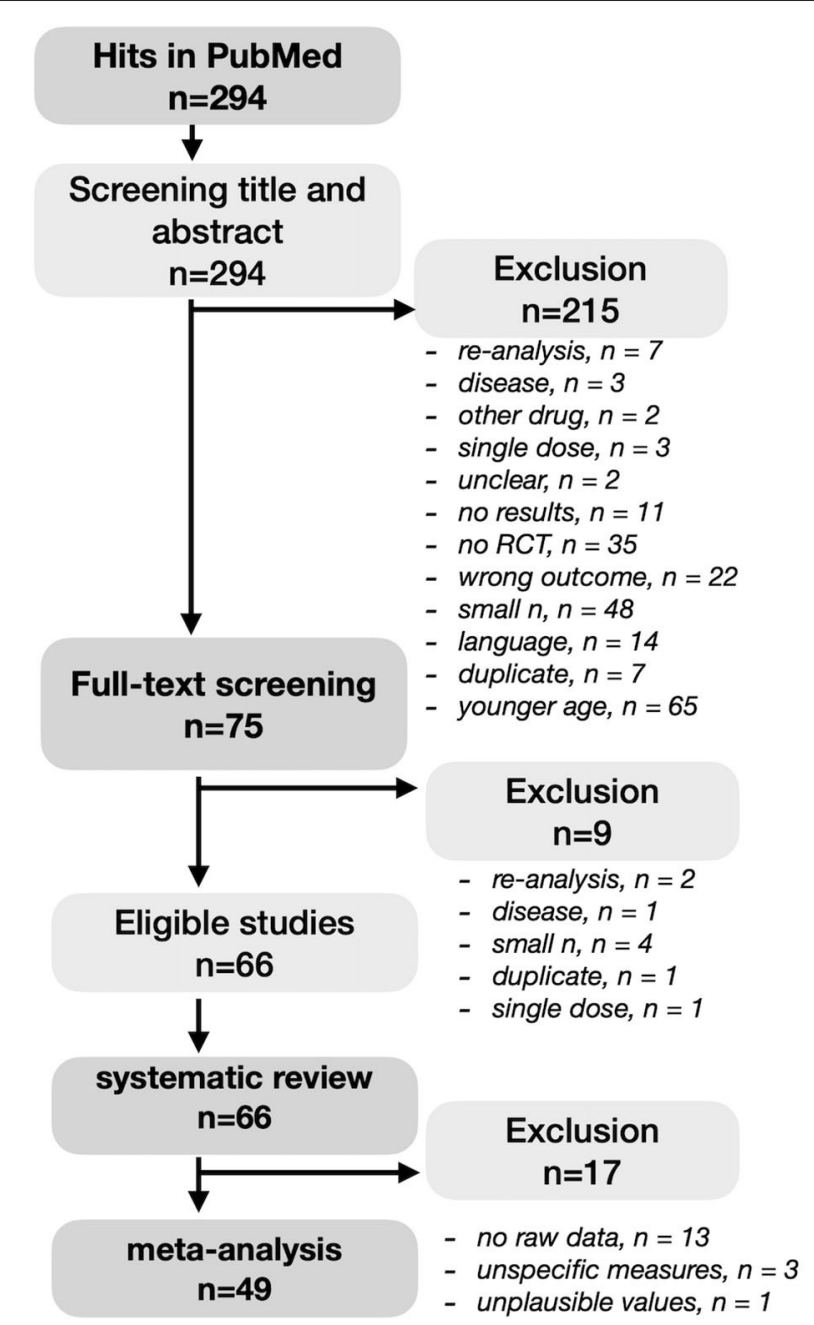

FIGURE 1 | PRISMA diagram of the systematic literature search.

number of minimally 20 participants per group was used to decrease the likelihood of a type II error, which can skew the results toward not finding a (small) effect that is truly there. Considering the age range definition, cognitive decline already starts from young adulthood, but by middle age, from around 40 , neuronal volume shrinkage in both white and gray matter becomes more apparent $(26,27)$. According title and abstract screening, as well as occasionally briefly consulting full-texts, resulted in 75 RCTs with the majority of studies excluded due to wrong population, wrong micronutrient, no memory measure, no RCT, younger age, small sample. During the full-text screening, another nine articles were excluded based on re-analysis $(n=2)$, non-dementia disease $(n=1)$, small sample size $(n=4)$, duplicate $(n=1)$, and single dose study $(n=1)$. Full-text screening eventually resulted in 66 articles that were included in the systematic review. Effect sizes could be derived or calculated from 49 articles and included in a meta-analysis. Due to unavailable raw value information, effect sizes could not be retrieved from 17 studies.
As all included articles provided relatively large sample sizes per group $(>n=20)$ and all were carefully checked for following a randomized trial design being regarded as the gold standard, we refrained from applying additional quality evaluation tools.

\section{Included Polyphenols and Their Effects on Memory}

The identified studies investigated either polyphenol-rich plant extracts such as berries, cherries, grapes, pomegranate, green tea, and Ginkgo biloba, cocoa flavanols, curcumin, Pinus radiata bark, spearmint extract, soy isoflavonol, or rather purified polyphenols such as resveratrol (see Table 1 for a more detailed overview). In addition, studies included different control conditions, either placebo or no treatment, or a lower dosage of the polyphenol treatment, or an alternative drug, such as rivastigmin or donezepil for treatment of Alzheimer's disease symptoms or alternative substances not containing polyphenols. Results were further reviewed according to these categories and reported WM and EM outcomes, respectively.

\section{Evaluation of Effect Sizes}

Effect size d for all studies with available data [mean, standard deviation (SD) or F-statistics] was computed in the following way: (1) for studies with available mean and SD for 1 timepoint for 2 groups, we calculated effect sizes using the "Means, Standard Deviations, and Sample Sizes" algorithm available at https://www.campbellcollaboration.org/escalc/html/ EffectSizeCalculator-SMD1.php, (2) for studies with mean and SD for 2 timepoints for 2 groups (repeated measure design), we calculated effect sizes for mean differences of groups with (un)equal sample size within a pre-post-control design using the algorithm at https://www.psychometrica.de/ effect_size.html, and (3) for studies with F-statistics only (no mean, SD available), we calculated effect size according to the algorithm at https://www.campbellcollaboration.org/escalc/ html/EffectSizeCalculator-SMD4.php. If necessary, standard error (SE) was transformed to SD by calculating $\mathrm{SD}=\mathrm{SE} \times$ $\operatorname{sqr}(n)$.

Effect size error $\mathrm{SE}_{\mathrm{d}}$ was computed according to:

$$
S E_{d}=\sqrt{\frac{n_{1}+n_{2}}{n_{1} n_{2}}+\frac{d^{2}}{2\left(n_{1}+n_{2}\right)}}
$$

with effect size (d), intervention sample $\left(\mathrm{n}_{1}\right)$ and control sample $\left(\mathrm{n}_{2}\right)$. For those memory outcomes, where higher scores relate to lower performance (i.e., reaction time), effect size valence was inversed, to represent memory improvements. At maximum four outcome measures (2 WM, 2 EM) were included in the metaanalysis per study due to feasibility. Effect sizes were calculated for the most sensitive measures (for WM e.g., Stroop inference reaction time and for EM e.g., delayed recall of a 10-15 words list) and in case of more than two groups or timepoints, for the group that had a single formula and/or the highest dose compared to placebo/lowest dose; and for the longest intervention period. A deviation of this rule was made for Wightman et al. (83) reporting effect sizes for (1) Sideritis scardica extract and (2) Ginkgo biloba compared to placebo. 
TABLE 1 | Details of the 66 included studies in the review.

\begin{tabular}{|c|c|c|c|c|c|c|c|c|c|c|c|c|c|c|c|}
\hline $\begin{array}{l}\text { Study } \\
\text { no.\# }\end{array}$ & First author & $\begin{array}{l}\text { Publication } \mathrm{J} \\
\text { year }\end{array}$ & Journal & Population & $\begin{array}{l}\text { Age } \\
\text { range } \\
\text { (years) }\end{array}$ & $\begin{array}{l}\text { Name of the } \\
\text { examined } \\
\text { extract, } \\
\text { polyphenol } \\
\text { or sublass } \\
\text { (referred to } \\
\text { as main or } \\
\text { effective } \\
\text { ingredient } \\
\text { by the } \\
\text { authors) }\end{array}$ & $\begin{array}{l}\text { Intervention, amount } \\
\text { of polyphenols }\end{array}$ & Control & $\begin{array}{l}\text { Duration of } \\
\text { intervention/ } \\
\text { control } \\
\text { period }\end{array}$ & $\mathbf{n}$ & $\begin{array}{l}\text { Cognitive tests } \\
\text { related to working } \\
\text { or episodic } \\
\text { memory } \\
\text { performance }\end{array}$ & $\begin{array}{l}\text { Any } \\
\text { significant } \\
\text { outcome }{ }^{\circ} \\
\text { none, + yes } \\
\text { positive; - } \\
\text { yes } \\
\text { negative) }\end{array}$ & $\begin{array}{l}\text { Significant } \\
\text { outcomes } \\
\text { in } \\
\text { working } \\
\text { (WM) or } \\
\text { episodic } \\
\text { memory } \\
\text { (EM), or } \\
\text { both }\end{array}$ & $\begin{array}{l}\text { included in } \\
\text { metaanalysis }\end{array}$ & Reference \\
\hline 1 & Ahles S & 2020 & Nutrients & $\begin{array}{l}\text { healthy adults } \\
\text { (overweight- } \\
\text { obese, } \\
\text { BMI25-35) }\end{array}$ & $40-60$ & anthocyanins & $\begin{array}{l}90 \mathrm{mg} \text { Aronia } \\
\text { melanocarpa, } 150 \mathrm{mg} \\
\text { Aronia melanocarpa } \\
\text { (18\%anthocyanins)/day }\end{array}$ & maltodextrin & 24 weeks & $\begin{array}{l}102(97 \\
\text { completed) }\end{array}$ & Stroop & 。 & & yes & (28) \\
\hline 2 & Basaria S & 2009 & $\begin{array}{l}J \\
\text { Endocrinol Invest }\end{array}$ & $\begin{array}{l}\text { perimenopausal } \\
\text { women }\end{array}$ & $46-76$ & isoflavones & $\begin{array}{l}160 \mathrm{mg} \text { of total } \\
\text { isoflavones ( } 96 \mathrm{mg} \\
\text { aglycones)/day }\end{array}$ & casein & 12 weeks & $\begin{array}{l}93 \text { ( } 84 \\
\text { completed, } \\
46+38)\end{array}$ & TMTB & $\circ$ & & $\begin{array}{l}\text { no (unplausible } \\
\text { values) }\end{array}$ & (29) \\
\hline 3 & Beck SM & 2016 & $\begin{array}{l}\text { Hum } \\
\text { Psychopharmacol }\end{array}$ & $\begin{array}{l}\text { healthy adults, } \\
\text { SMl }\end{array}$ & $50-65$ & ginkgo biloba & $\begin{array}{l}240 \mathrm{mg}, 22-27 \% \\
\text { ginkgo flavones/day }\end{array}$ & $\begin{array}{l}\text { placebo } \\
\text { (unclear) }\end{array}$ & 8 weeks & $\begin{array}{l}75 \text { (61-30,31 } \\
\text { completed) }\end{array}$ & $\begin{array}{l}\text { task-set switching, } \\
\text { delayed response } \\
\text { task, } \\
\text { prospective memory }\end{array}$ & + & WM & yes & (30) \\
\hline 4 & Bensalem J & 2019 & $\begin{array}{l}\text { J Gerontol A Biol } \\
\text { Sci Med Sci }\end{array}$ & healthy adults & $60-70$ & anthocyanins & $600 \mathrm{mg} / \mathrm{day}$ & $\begin{array}{l}\text { placebo } \\
\text { (unclear) }\end{array}$ & 24 weeks & $\begin{array}{l}215 \text { (190 } \\
\text { completed) }\end{array}$ & $\begin{array}{l}\text { Cambridge } \\
\text { Neuropsychological } \\
\text { Test Automated } \\
\text { Battery (CANTAB): } \\
\text { PALTEA, VRMFR, } \\
\text { VRMR, SSP, } \\
\text { reverse SSP }\end{array}$ & + & EM & yes & (31) \\
\hline 5 & Burns NR & 2006 & $\begin{array}{l}\text { Hum } \\
\text { Psychopharmacol }\end{array}$ & healthy adults & $55-79$ & ginkgo biloba & $120 \mathrm{mg} /$ day & $\begin{array}{l}\text { placebo } \\
\text { (unclear) }\end{array}$ & 12 weeks & 93 & $\begin{array}{l}\text { test battery including } \\
\text { wordlist, } \\
\text { Stroop, digit span }\end{array}$ & + & EM & yes & (32) \\
\hline 6 & Carlson JJ & 2007 & J Am Diet Assoc & healthy adults & $65-84$ & ginkgo biloba & $\begin{array}{l}3 \text { xcapsules, =160 mg } \\
\text { ginkgo biloba, } 68 \mathrm{mg} \\
\text { gotu kola, and } 180 \mathrm{mg} \\
\text { decosahexaenoic acid, } \\
\text { bioflavonoid } \\
\text { concentrate (100 mg) } \\
\text { and vitamin A } \\
\text { (300 IU)/day }\end{array}$ & $\begin{array}{l}\text { identical } \\
\text { capsules } \\
\text { (gelatin, } \\
\text { glycerin, } \\
\text { soybean oil, } \\
\text { yellow } \\
\text { beeswax, } \\
\text { lecithin, corn } \\
\text { oil, natural } \\
\text { caramel color, } \\
\text { and } \\
\text { maltodextrin) }\end{array}$ & 4 months & $\begin{array}{l}90 \text { (78 } \\
\text { completed) }\end{array}$ & $\begin{array}{l}\text { benton visual } \\
\text { retention, word } \\
\text { list learning }\end{array}$ & - & EM & yes & (33) \\
\hline 7 & Casini ML & 2006 & Fertil Steril & $\begin{array}{l}\text { postmenopausal } \\
\text { women }\end{array}$ & $\begin{array}{l}>12 \\
\text { months } \\
\text { after } \\
\text { menses } \\
\text { absence }\end{array}$ & isoflavones & $\begin{array}{l}600 \mathrm{mg} / \text { day }(60 \mathrm{mg} / \mathrm{day} \\
\text { isoflavones) }\end{array}$ & $\begin{array}{l}\text { identical } \\
\text { placebo } \\
\text { (unclear) }\end{array}$ & 6 months & $\begin{array}{l}78 \text { (76 } \\
\text { completed, } \\
\text { cross-over) }\end{array}$ & digit span & + & WM & yes & (34) \\
\hline 8 & Cieza A & 2003 & Fortschr Med Orig & healthy adults & $50-65$ & ginkgo biloba & $240 \mathrm{mg} / \mathrm{day}$ & $\begin{array}{l}\text { placebo } \\
\text { (unclear) }\end{array}$ & 4 weeks & $\begin{array}{l}66 \text { (66 } \\
\text { completed) }\end{array}$ & $\begin{array}{l}\text { different } \\
\text { psychological tests } \\
\text { including Stroop, } \\
\text { digit span }\end{array}$ & $\circ$ & & yes & (35) \\
\hline
\end{tabular}


TABLE 1 | Continued

\begin{tabular}{|c|c|c|c|c|c|c|c|c|c|c|c|c|c|c|c|}
\hline $\begin{array}{l}\text { Study } \\
\text { no.\# }\end{array}$ & ly First author & $\begin{array}{l}\text { Publication } \\
\text { year }\end{array}$ & Journal & Population & $\begin{array}{l}\text { Age } \\
\text { range } \\
\text { (years) }\end{array}$ & $\begin{array}{l}\text { Name of the } \\
\text { examined } \\
\text { extract, } \\
\text { polyphenol } \\
\text { or sublass } \\
\text { (referred to } \\
\text { as main or } \\
\text { effective } \\
\text { ingredient } \\
\text { by the } \\
\text { authors) }\end{array}$ & $\begin{array}{l}\text { Intervention, amount } \\
\text { of polyphenols }\end{array}$ & Control & $\begin{array}{l}\text { Duration of } \\
\text { intervention/ } \\
\text { control } \\
\text { period }\end{array}$ & $\mathrm{n}$ & $\begin{array}{l}\text { Cognitive tests } \\
\text { related to working } \\
\text { or episodic } \\
\text { memory } \\
\text { performance }\end{array}$ & $\begin{array}{l}\text { Any } \\
\text { significant } \\
\text { outcome }{ }^{\circ} \\
\text { none, + yes } \\
\text { positive; - } \\
\text { yes } \\
\text { negative) }\end{array}$ & $\begin{array}{l}\text { Significant } \\
\text { outcomes } \\
\text { in } \\
\text { working } \\
\text { (WM) or } \\
\text { episodic } \\
\text { memory } \\
\text { (EM), or } \\
\text { both }\end{array}$ & $\begin{array}{l}t \text { included in } \\
\text { metaanalysis }\end{array}$ & Reference \\
\hline 9 & Cox KH & 2015 & $\begin{array}{l}\text { J } \\
\text { Psychopharmacol }\end{array}$ & healthy adults & 60-85 & curcuminoids & $\begin{array}{l}400 \mathrm{mg} / \text { day } \\
\text { Longvida® } \\
\text { Optimized Curcumin } \\
\text { (containing } \\
\text { approximately } 80 \\
\text { mg curcumin) }\end{array}$ & $\begin{array}{l}\text { dextrin }+ \\
\text { yellow } \\
\text { food powder }\end{array}$ & 4 weeks & $\begin{array}{l}61 \text { ( } 60 \\
\text { completed) }\end{array}$ & $\begin{array}{l}\text { parallel versions of } \\
\text { tasks from the } \\
\text { Computerised } \\
\text { Mental Performance } \\
\text { Assessment System }\end{array}$ & + & WM & yes & (18) \\
\hline 10 & Cox KHM & 2020 & Nutrients & healthy adults & $50-80$ & curcuminoids & $\begin{array}{l}\text { 400mg/day Longvida } \\
\text { (80mg/day curcumin) }\end{array}$ & $\begin{array}{l}\text { dextrin }+ \\
\text { yellow } \\
\text { food powder }\end{array}$ & 12 weeks & $\begin{array}{l}89 \text { ( } 79 \\
\text { completed) }\end{array}$ & $\begin{array}{l}\text { Serial substractions, } \\
\text { Virtual Morris Water } \\
\text { Maze (VMWM) }\end{array}$ & + & WM & yes & (36) \\
\hline 11 & Desideri G & 2012 & Hypertension & $\mathrm{MCl}$ & $64-82$ & $\begin{array}{l}\text { cocoa } \\
\text { flavanols }\end{array}$ & $\begin{array}{l}\text { high (HF: } \approx 990 \mathrm{mg} \\
\text { flavanols/day) or } \\
\text { intermediate (IF: } \approx 520 \\
\text { mg flavanols/day }\end{array}$ & $\begin{array}{l}\text { low level (LF: } \\
\approx 48 \mathrm{mg} \\
\text { flavanols/day) }\end{array}$ & 8 weeks & 90 & $\begin{array}{l}\text { Verbal } \\
\text { fluency, TMTA, } \\
\text { TMTB }\end{array}$ & + & WM & yes & (37) \\
\hline 12 & Dodge $\mathrm{HH}$ & 2008 & Neurology & healthy adults & $>85$ & ginkgo biloba & $\begin{array}{l}3 \times 80 \mathrm{mg},=240 \mathrm{mg} / \text { day } \\
(24 \% \text { flavone } \\
\text { glycosides) }\end{array}$ & unclear & 42 months & $\begin{array}{l}134 \text { (118 } \\
\text { completed) }\end{array}$ & $\begin{array}{l}\text { Cerad } \\
\text { verbal learning }\end{array}$ & 。 & & $\begin{array}{l}\text { no (no raw } \\
\text { measures } \\
\text { available) }\end{array}$ & (38) \\
\hline 13 & Evans HM & 2017 & Nutrients & $\begin{array}{l}\text { cognitively intact } \\
\text { post- } \\
\text { menopausal } \\
\text { women }\end{array}$ & $45-85$ & resveratrol & $150 \mathrm{mg} /$ day & $\begin{array}{l}\text { placebo } \\
\text { (unclear) }\end{array}$ & 14 weeks & $\begin{array}{l}80 \text { (72 } \\
\text { completed) }\end{array}$ & $\begin{array}{l}\text { Cambridge Semantic } \\
\text { Memory } \\
\text { Battery; Rey } \\
\text { Auditory Verbal } \\
\text { Learning Test } \\
\text { (RAVLT; Double } \\
\text { Span Task }\end{array}$ & + & EM & yes & (39) \\
\hline 14 & Fournier LR & 2007 & $\begin{array}{l}\text { J Nutr } \\
\text { Health Aging }\end{array}$ & $\begin{array}{l}\text { postmenopausal } \\
\text { women }\end{array}$ & $48-65$ & isoflavones & $\begin{array}{l}\text { soy milk or soy } \\
\text { supplement } \\
\text { (70/72mg isoflavones) }\end{array}$ & $\begin{array}{l}\text { cow milk, } \\
\text { placebo supp. }\end{array}$ & 16 weeks & 79 & $\begin{array}{l}\text { Stroop, pattern } \\
\text { recognition, } \\
\text { benton, etc. }\end{array}$ & - & WM & $\begin{array}{l}\text { no (no raw } \\
\text { measures } \\
\text { available) }\end{array}$ & (40) \\
\hline 15 & Furlong ON & 2020 & Eur $\mathrm{J}$ Nutr & $\begin{array}{l}\text { postmenopausal } \\
\text { women }\end{array}$ & $44-63$ & isoflavones & $\begin{array}{l}60 \mathrm{mg} / \text { day or } \\
35 \mathrm{mg} / \text { day (in } 350 \mathrm{ml} \\
\text { soy drink) }\end{array}$ & $10 \mathrm{mg} / \mathrm{day}$ & 12 weeks & $\begin{array}{l}115 \text { (101 } \\
\text { analysed) }\end{array}$ & $\begin{array}{l}\text { spatial working } \\
\text { memory, } \\
\text { spatial span, pattern } \\
\text { recognition memory, } \\
\text { 5-choice reaction } \\
\text { time, and match to } \\
\text { sample visual search }\end{array}$ & 。 & & yes & (41) \\
\hline 16 & Gavrilova SI & 2014 & $\begin{array}{l}\text { Int J Geriatr } \\
\text { Psychiatry }\end{array}$ & $\mathrm{MCl}$ & $>55$ & ginkgo biloba & $240 \mathrm{mg}$ per day & $\begin{array}{l}\text { placebo } \\
\text { (unclear) }\end{array}$ & 24 weeks & $\begin{array}{l}160(145 \\
\text { analysed) }\end{array}$ & TMT-B & + & WM & yes & (42) \\
\hline 17 & Gleason CE & 2015 & J Alzheimers Dis & $A D$ & $>60$ & isoflavones & $100 \mathrm{mg} / \mathrm{day}$ & maltodextrin & 6 months & $\begin{array}{l}65 \text { (59 } \\
\text { completed) }\end{array}$ & $\begin{array}{l}\text { verbal and } \\
\text { visuospatial memory, } \\
\text { Benton retention } \\
\text { task, figure recall, } \\
\text { TMT, etc) }\end{array}$ & 。 & & yes & (43) \\
\hline 18 & Hartley DE & 2004 & Nutr Neurosci & $\begin{array}{l}\text { postmenopausal } \\
\text { women }\end{array}$ & $51-66$ & ginkgo biloba & $\begin{array}{l}320 \mathrm{mg} \text { Gincosan/day } \\
\text { (120mg ginkgo }+ \\
200 \mathrm{mg} \text { ginseng) }\end{array}$ & unclear & 12 weeks & $\begin{array}{l}70 \text { ( } 57 \\
\text { completed) }\end{array}$ & $\begin{array}{l}\text { verbal and visual } \\
\text { memory, CANTAB, } \\
\text { delayed matching to } \\
\text { sample test }\end{array}$ & 。 & & yes & (44) \\
\hline
\end{tabular}


TABLE 1 | Continued

\begin{tabular}{|c|c|c|c|c|c|c|c|c|c|c|c|c|c|c|c|}
\hline $\begin{array}{l}\text { Study } \\
\text { no.\# }\end{array}$ & y First author & $\begin{array}{l}\text { Publication } \\
\text { year }\end{array}$ & Journal & Population & $\begin{array}{l}\text { Age } \\
\text { range } \\
\text { (years) }\end{array}$ & $\begin{array}{l}\text { Name of the } \\
\text { examined } \\
\text { extract, } \\
\text { polyphenol } \\
\text { or sublass } \\
\text { (referred to } \\
\text { as main or } \\
\text { effective } \\
\text { ingredient } \\
\text { by the } \\
\text { authors) }\end{array}$ & $\begin{array}{l}\text { Intervention, amount } \\
\text { of polyphenols }\end{array}$ & Control & $\begin{array}{l}\text { Duration of } \\
\text { intervention/ } \\
\text { control } \\
\text { period }\end{array}$ & $\mathrm{n}$ & $\begin{array}{l}\text { Cognitive tests } \\
\text { related to working } \\
\text { or episodic } \\
\text { memory } \\
\text { performance }\end{array}$ & $\begin{array}{l}\text { Any } \\
\text { significant } \\
\text { outcome }\left(^{\circ}\right. \\
\text { none, + yes } \\
\text { positive; - } \\
\text { yes } \\
\text { negative) }\end{array}$ & $\begin{array}{l}\text { Significant } \\
\text { outcomes } \\
\text { in } \\
\text { working } \\
\text { (WM) or } \\
\text { episodic } \\
\text { memory } \\
\text { (EM), or } \\
\text { both }\end{array}$ & $\begin{array}{l}t \text { included in } \\
\text { metaanalysis }\end{array}$ & Reference \\
\hline 19 & $\begin{array}{l}\text { Henderson } \\
\text { WW }\end{array}$ & 2012 & Neurology & $\begin{array}{l}\text { perimenopausal } \\
\text { women }\end{array}$ & $\begin{array}{l}45-92 \\
\text { years }\end{array}$ & isoflavones & $\begin{array}{l}25 \mathrm{~g} / \text { day of isoflavone } \\
\text { rich soy } \\
\text { protein(contained } \\
91 \mathrm{mg} \text { aglycone weight } \\
\text { isoflavones ( } 154 \mathrm{mg} \\
\text { total isoflavone } \\
\text { equivalents) composed } \\
\text { of genistein ( } 52 \mathrm{mg} \\
\text { aglycone equivalents), } \\
\text { daidzein (36 mg } \\
\text { aglycone equivalents), } \\
\text { and gly- citein ( } 3 \mathrm{mg} \\
\text { aglycone equivalents)) }\end{array}$ & $\begin{array}{l}\text { milk protein } \\
\text { placebo }\end{array}$ & 2.5 years & $\begin{array}{l}350 \text { (313 } \\
\text { completed) }\end{array}$ & $\begin{array}{l}\text { Immediate recall, } \\
\text { faces } 2 \text { delayed } \\
\text { recall }\end{array}$ & + & EM & yes & (24) \\
\hline 20 & Herrlinger KA & 2018 & $\begin{array}{l}J \text { Altern } \\
\text { Complement Med }\end{array}$ & healthy adults & $50-70$ & $\begin{array}{l}\text { chlorogenic } \\
\text { acid }\end{array}$ & $\begin{array}{l}900 \mathrm{mg} \text { or } 600 \mathrm{mg} \\
\text { spearmint extract/day }\end{array}$ & $\begin{array}{l}\text { powder with } 0 \\
\text { mg/ day } \\
\text { spearmint } \\
\text { extract }\end{array}$ & 90 days & $\begin{array}{l}90(87 \\
\text { completed) }\end{array}$ & CDR battery & + & WM & yes & (45) \\
\hline 21 & Herrschaft $\mathrm{H}$ & 2012 & J Psychiatr Res & $\begin{array}{l}\text { mild-moderate } \\
\mathrm{AD}\end{array}$ & $>50$ & ginkgo biloba & $240 \mathrm{mg} / \mathrm{day}$ & unclear & 24 weeks & $\begin{array}{l}410(404 \\
\text { analysed) }\end{array}$ & $\begin{array}{l}\text { SKT, CGIC, verbal } \\
\text { fluency }\end{array}$ & + & WM & $\begin{array}{l}\text { no (no raw } \\
\text { measures } \\
\text { available) }\end{array}$ & (46) \\
\hline 22 & Ho SC & 2007 & Menopause & $\begin{array}{l}\text { postmenopausal } \\
\text { women }\end{array}$ & $55-76$ & isoflavones & $\begin{array}{l}80 \mathrm{mg} / \text { day soy-derived } \\
\text { isoflavones }\end{array}$ & $\begin{array}{l}\text { identical } \\
\text { appearing } \\
\text { placebo }\end{array}$ & 6 months & $\begin{array}{l}200 \text { (data } \\
\text { analyse van } \\
176)\end{array}$ & $\begin{array}{l}\text { the Hong Kong List } \\
\text { Learning Test= Tests } \\
\text { of learning and } \\
\text { memory (assesses } \\
\text { rate of learning, rate } \\
\text { of forgetting, } \\
\text { encoding and } \\
\text { retrieval deficits, and } \\
\text { learning strategies) \& } \\
\text { Rey- Osterrieth } \\
\text { Complex Figure Test } \\
\text { and Wechsler } \\
\text { Memory } \\
\text { Scale-Revised = } \\
\text { visuospatial } \\
\text { constructional ability } \\
\text { and visual memory }\end{array}$ & 。 & & yes & (47) \\
\hline 23 & Huhn S & 2018 & Neuroimage & healthy adults & $60-78$ & resveratrol & $\begin{array}{l}200 \mathrm{mg} / \text { day of } \\
\text { resveratrol and } 320 \mathrm{mg} \\
\text { of quercetin }\end{array}$ & $\begin{array}{l}\text { placebo } \\
\text { (identical } \\
\text { w/o } \\
\text { resveratrol) }\end{array}$ & 26 weeks & 60 (53 over) & $\begin{array}{l}\text { California Verbal } \\
\text { Learning Task (CVLT, } \\
\text { main outcome), the } \\
\text { ModBent task, }\end{array}$ & 。 & & yes & (48) \\
\hline
\end{tabular}




\begin{tabular}{|c|c|c|c|c|c|c|c|c|c|c|c|c|c|c|c|}
\hline $\begin{array}{l}\text { Study } \\
\text { no.\# }\end{array}$ & First author & $\begin{array}{l}\text { Publication } \\
\text { year }\end{array}$ & Journal & Population & $\begin{array}{l}\text { Age } \\
\text { range } \\
\text { (years) }\end{array}$ & $\begin{array}{l}\text { Name of the } \\
\text { examined } \\
\text { extract, } \\
\text { polyphenol } \\
\text { or sublass } \\
\text { (referred to } \\
\text { as main or } \\
\text { effective } \\
\text { ingredient } \\
\text { by the } \\
\text { authors) }\end{array}$ & $\begin{array}{l}\text { Intervention, amount } \\
\text { of polyphenols }\end{array}$ & Control & $\begin{array}{l}\text { Duration of } \\
\text { intervention/ } \\
\text { control } \\
\text { period }\end{array}$ & $\mathbf{n}$ & $\begin{array}{l}\text { Cognitive tests } \\
\text { related to working } \\
\text { or episodic } \\
\text { memory } \\
\text { performance }\end{array}$ & $\begin{array}{l}\text { Any } \\
\text { significant } \\
\text { outcome }{ }^{\circ} \\
\text { none, + yes } \\
\text { positive; - } \\
\text { yes } \\
\text { negative) }\end{array}$ & $\begin{array}{l}\text { Significant } \\
\text { outcomes } \\
\text { in } \\
\text { working } \\
\text { (WM) or } \\
\text { episodic } \\
\text { memory } \\
\text { (EM), or } \\
\text { both }\end{array}$ & $\begin{array}{l}t \text { included in } \\
\text { metaanalysis }\end{array}$ & Reference \\
\hline 24 & Jackson PA & 2016 & Nutrients & $\begin{array}{l}\text { healthy adults, } \\
\text { SMI }\end{array}$ & $55-65$ & ginkgo biloba & Ginkgo biloba (240 mg) & $\begin{array}{l}2.24 \mathrm{~g} \text { high } \\
\text { oleic acid } \\
\text { sunflower oil } \\
\text { and } 120 \mathrm{mg} \\
\text { fish oil } \\
(32 \mathrm{mg} \text { DHA } \\
+ \text { EPA) }\end{array}$ & 24 weeks & $\begin{array}{l}248 \text { ( } 84 \\
\text { completed) }\end{array}$ & $\begin{array}{l}\text { Cognitive Demand } \\
\text { Battery(CDB), Rapid } \\
\text { Visual Information } \\
\text { Processing(RVIP) }\end{array}$ & 。 & & yes & (49) \\
\hline 25 & Kanowski S & 1996 & $\begin{array}{l}\text { Pharmacopsychiatr } \\
\text { y }\end{array}$ & $\begin{array}{l}\text { AD or } \\
\text { multi-infarct } \\
\text { dementia }\end{array}$ & $>55$ & ginkgo biloba & $\begin{array}{l}240 \text { mg EGb } 761 \AA \text {, } \\
22-27 \% \text { Ginkgo } \\
\text { flavonooids, 2.8-3.4\% } \\
\text { ginkgolides A, B, C, } \\
2.6-3.2 \% \text { bilobalide }\end{array}$ & $\begin{array}{l}\text { placebo } \\
\text { (unclear) }\end{array}$ & 24 weeks & $\begin{array}{l}216(156 / 205 \\
\text { analysed) }\end{array}$ & CGI, SKT, NAB & + & WM & $\begin{array}{l}\text { no (no raw } \\
\text { measures } \\
\text { available) }\end{array}$ & (50) \\
\hline 26 & Kaschel R & 2011 & Phytomedicine & healthy adults & $45-65$ & ginkgo biloba & $\begin{array}{l}240 \text { mg EGb } 761 \AA \text {, } \\
22-27 \% \text { Ginkgo } \\
\text { flavonoids, 2.8-3.4\% } \\
\text { ginkgolides A, B, C, } \\
2.6-3.2 \% \text { bilobalide }\end{array}$ & $\begin{array}{l}\text { unclear, } \\
\text { placebo } \\
\text { tablets }\end{array}$ & 6 weeks & $\begin{array}{l}188 \text { (177 for } \\
\text { Memory } \\
\text { tasks) }\end{array}$ & $\begin{array}{l}\text { standardised free } \\
\text { recall paradigm, } \\
\text { standardised } \\
\text { recognition test }\end{array}$ & + & EM & yes & (51) \\
\hline 27 & Kean RJ & 2015 & Am J Clin Nutr & healthy adults & $60-81$ & flavonoids & $\begin{array}{l}549 \mathrm{mg} \text { hesperidin/L, } \\
60 \mathrm{mg} \\
\text { narirutin/L(250 mL } \\
\text { twice per day) }\end{array}$ & $\begin{array}{l}\text { low flavone } \\
\text { drink }\end{array}$ & 8 weeks & $\begin{array}{l}\text { 35-28 } \\
\text { (cross-over) }\end{array}$ & $\begin{array}{l}\text { Spatial Working } \\
\text { Memory }\end{array}$ & + & EM & yes & (52) \\
\hline 28 & Kent K & 2017 & Eur J Nutr & $\begin{array}{l}\text { mild-moderate } \\
A D\end{array}$ & $>70$ & anthocyanins & $\begin{array}{l}200 \mathrm{ml} / \text { day of cherry } \\
\text { juice }\end{array}$ & $\begin{array}{l}\text { control juice } \\
\text { with } \\
\text { negligible } \\
\text { amounts } \\
\text { of } \\
\text { anthocyanins }\end{array}$ & 12 weeks & 49 & $\begin{array}{l}\text { battery of seven } \\
\text { cognitive tests: } \\
\text { RAVLT, SOPT, } \\
\text { Boston naming test, } \\
\text { TMT(A and B), digit } \\
\text { span backwards } \\
\text { task, category verbal } \\
\text { fluency and letter } \\
\text { verbal fluency }\end{array}$ & + & EM & yes & (13) \\
\hline 29 & $\begin{array}{l}\text { Kreijkamp- } \\
\text { Kaspers S }\end{array}$ & 2004 & JAMA & $\begin{array}{l}\text { postmenopausal } \\
\text { women }\end{array}$ & $60-75$ & isoflavones & $\begin{array}{l}25.6 \mathrm{~g} / \text { day of soy } \\
\text { protein (containing } \\
99 \mathrm{mg} \text { of isoflavones: } \\
52 \mathrm{mg} \text { genistein, } 41 \\
\mathrm{mg} \text { daidzein, and } 6 \mathrm{mg} \\
\text { glycetein) }\end{array}$ & milk protein & 12 months & $\begin{array}{l}202(175 \\
\text { completed) }\end{array}$ & $\begin{array}{l}\text { Rey auditory verbal } \\
\text { learning test, } \\
\text { measures of short- } \\
\text { term and long-term } \\
\text { verbal and visual } \\
\text { memory, Doors test, } \\
\text { Digit span test }\end{array}$ & $\circ$ & & yes & (53) \\
\hline 30 & $\begin{array}{l}\text { Kritz- } \\
\text { Silverstein } \\
\text { D }\end{array}$ & 2003 & Menopause & healthy adults & $55-74$ & isoflavones & $\begin{array}{l}110 \mathrm{mg} / \text { day total } \\
\text { isoflavones }\end{array}$ & $\begin{array}{l}\text { identical } \\
\text { placebo }\end{array}$ & 6 months & $\begin{array}{l}56 \text { (53 } \\
\text { completed) }\end{array}$ & $\begin{array}{l}\text { WMS Logical } \\
\text { Memory and Recall }\end{array}$ & 。 & & yes & (54) \\
\hline 31 & $\begin{array}{l}\text { Kuszewski } \\
\text { JC }\end{array}$ & 2020 & J Nutr & healthy adults & $50-80$ & curcuminoids & $\begin{array}{l}160 \mathrm{mg} / \text { day curcumin } \\
\text { or } 160 \mathrm{mg} / \text { day } \\
\text { combined with fish oil } \\
\text { supplementation (2000 } \\
\mathrm{mg} / \mathrm{d} \text { DHA }+400 \\
\mathrm{mg} / \mathrm{day} \text { EPA) }\end{array}$ & $\begin{array}{l}\text { fish oil } 1 \\
\text { supplementation } \\
(2000 \mathrm{mg} / \mathrm{d} \\
\mathrm{DHA}+ \\
400 \mathrm{mg} / \mathrm{day} \\
\text { EPA) }\end{array}$ & 16 weeks & $\begin{array}{l}152(134 \\
\text { completed, } \\
126 \text { analysed) }\end{array}$ & $\begin{array}{l}\text { neuropsychological } \\
\text { test battery }\end{array}$ & $\circ$ & & $\begin{array}{l}\text { no (no raw } \\
\text { measures } \\
\text { available) }\end{array}$ & (55) \\
\hline
\end{tabular}


TABLE 1 | Continued

\begin{tabular}{|c|c|c|c|c|c|c|c|c|c|c|c|c|c|c|c|}
\hline $\begin{array}{l}\text { Study } \\
\text { no.\# }\end{array}$ & First author & $\begin{array}{l}\text { Publication } \\
\text { year }\end{array}$ & Journal & Population & $\begin{array}{l}\text { Age } \\
\text { range } \\
\text { (years) }\end{array}$ & $\begin{array}{l}\text { Name of the } \\
\text { examined } \\
\text { extract, } \\
\text { polyphenol } \\
\text { or sublass } \\
\text { (referred to } \\
\text { as main or } \\
\text { effective } \\
\text { ingredient } \\
\text { by the } \\
\text { authors) }\end{array}$ & $\begin{array}{l}\text { Intervention, amount } \\
\text { of polyphenols }\end{array}$ & Control & $\begin{array}{l}\text { Duration of } \\
\text { intervention/ } \\
\text { control } \\
\text { period }\end{array}$ & $\mathrm{n}$ & $\begin{array}{l}\text { Cognitive tests } \\
\text { related to working } \\
\text { or episodic } \\
\text { memory } \\
\text { performance }\end{array}$ & $\begin{array}{l}\text { Any } \\
\text { significant } \\
\text { outcome }{ }^{\circ} \\
\text { none, + yes } \\
\text { positive; - } \\
\text { yes } \\
\text { negative) }\end{array}$ & $\begin{array}{l}\text { Significant } \\
\text { outcomes } \\
\text { in } \\
\text { working } \\
\text { (WM) or } \\
\text { episodic } \\
\text { memory } \\
\text { (EM), or } \\
\text { both }\end{array}$ & $\begin{array}{l}t \text { included in } \\
\text { metaanalysis }\end{array}$ & Reference \\
\hline 32 & Le Bars PL & 1997 & JAMA & dementia & $>45$ & ginkgo biloba & $120 \mathrm{mg}$ ginkgo & $\begin{array}{l}\text { placebo (not } \\
\text { specified) }\end{array}$ & 52 weeks & $\begin{array}{l}309 \text { (202 } \\
\text { completed) }\end{array}$ & ADAS-cog & + & WM & $\begin{array}{l}\text { no (no raw } \\
\text { measures } \\
\text { available) }\end{array}$ & (56) \\
\hline 33 & Lewis JE & 2014 & $\begin{array}{l}\text { BMC Complement } \\
\text { Altern Med }\end{array}$ & healthy adults & $>60$ & ginkgo biloba & $\begin{array}{l}\text { 120mg ginkgo leaf, } \\
80 \mathrm{mg} \text { whole gingko } \\
\text { +others, +either } \\
700 \mathrm{mg} \text { choline vs OR } \\
\text { anthocyanins mixture, } \\
\text { green tea, vitamins etc. }\end{array}$ & $\begin{array}{l}\text { cellulose, } \\
\text { lactose, beet } \\
\text { powder }\end{array}$ & 6 months & $\begin{array}{l}97 \\
(33+31+33)\end{array}$ & TMT, Stroop & + & WM & $\begin{array}{l}\text { no (no raw } \\
\text { measures } \\
\text { available) }\end{array}$ & (57) \\
\hline 34 & LiS & 2019 & $J$ Int Med Res & $\mathrm{VaMCl}$ & $\begin{array}{l}\text { mean age } \\
65\end{array}$ & ginkgo biloba & $3 \times 19.2 \mathrm{mg}$ ginkgo & $\begin{array}{l}3 \times 1.8 \mathrm{mg} \\
\text { pushen } \\
\text { (traditional } \\
\text { Chinese } \\
\text { medicine) }\end{array}$ & 12 weeks & $\begin{array}{l}64 \text { (57 } \\
\text { completed) }\end{array}$ & MoCA & 。 & & yes & (58) \\
\hline 35 & $\begin{array}{l}\text { Mastroiacovo } \\
\text { D }\end{array}$ & 2015 & Am J Clin Nutr & $\begin{array}{l}\text { cognitively intact } \\
\text { adults }\end{array}$ & $61-85$ & $\begin{array}{l}\text { cocoa } \\
\text { flavanols }\end{array}$ & $\begin{array}{l}\text { drink containing } 993 \\
\mathrm{mg} / \text { day [high flavanol } \\
\text { (HF)], } 520 \mathrm{mg} \\
\text { [intermediate flavanol } \\
\text { (IF)] }\end{array}$ & $\begin{array}{l}48 \mathrm{mg} \text { [low } \\
\text { flavanol } \\
\text { (LF)] cocoa } \\
\text { flavanols (CFs) }\end{array}$ & 8 weeks & 90 & Verbal fluency test & + & WM & yes & (59) \\
\hline 36 & Mazza M & 2006 & Eur J Neurol & $\begin{array}{l}\text { mild-moderate } \\
\mathrm{AD}\end{array}$ & $50-80$ & ginkgo biloba & $\begin{array}{l}\text { daily: Ginkgo biloba: } \\
160 \mathrm{mg}\end{array}$ & $\begin{array}{l}\text { cholinesterase } \\
\text { inhibitor: } \\
\text { donepezil } \\
\text { ( } 5 \mathrm{mg} \text { daily } \\
\text { dose), } \\
\text { placebo }\end{array}$ & 24 weeks & $\begin{array}{l}74 \text { (60 } \\
\text { completed) }\end{array}$ & $\begin{array}{l}\text { SKT - Syndrome } \\
\text { Kurz test } \\
\text { (psychometric test } \\
\text { battery for } \\
\text { assessment of } \\
\text { memory and } \\
\text { attention), MMSE, } \\
\text { CGI }\end{array}$ & + & WM & $\begin{array}{l}\text { no (unspecific } \\
\text { outcome) }\end{array}$ & (60) \\
\hline 37 & McCarney R & 2008 & $\begin{array}{l}\text { Int J Geriatr } \\
\text { Psychiatry }\end{array}$ & dementia & $>50$ & ginkgo biloba & $\begin{array}{l}120 \mathrm{mg} \text { daily (60mg } \\
\text { twice a day); placebo: } \\
\text { lactose-based }\end{array}$ & $\begin{array}{l}\text { lactose-based } \\
\text { placebo }\end{array}$ & 6 months & $\begin{array}{l}176(176 \\
\text { analysed) }\end{array}$ & ADAS-cog & 。 & & $\begin{array}{l}\text { no (unspecific } \\
\text { outcome) }\end{array}$ & (61) \\
\hline 38 & $\begin{array}{l}\text { McNamara } \\
\text { RK }\end{array}$ & 2018 & Neurobiol Aging & SMI (mild) & $62-80$ & anthocyanins & $\begin{array}{l}\text { berry powder } 25 \mathrm{~g} \text { dry } \\
\text { weight, or combined } \\
\text { fish oil + blueberry }\end{array}$ & $\begin{array}{l}\text { placebo } \\
\text { isocaloric } \\
\text { carbohydrates } \\
\text { or } \\
\text { fish oil }\end{array}$ & 24 weeks & $\begin{array}{l}94 \\
\text { (21,24,26,23; } \\
76 \text { analysed) }\end{array}$ & $\begin{array}{l}\text { TMT, verbal learning, } \\
\text { fluency }\end{array}$ & 。 & & $\begin{array}{l}\text { no (no raw } \\
\text { measures } \\
\text { available) }\end{array}$ & (62) \\
\hline 39 & Mix JA & 2002 & $\begin{array}{l}\text { Hum } \\
\text { Psychopharmacol }\end{array}$ & $\begin{array}{l}\text { cognitively intact } \\
\text { adults }\end{array}$ & $>60$ & ginkgo biloba & $\begin{array}{l}180 \mathrm{mg} / \text { day of Ginkgo } \\
\text { biloba extract }\end{array}$ & $\begin{array}{l}\text { placebo } \\
\text { (unclear) }\end{array}$ & 6 weeks & $\begin{array}{l}262 \text { (149 } \\
\text { analysed) }\end{array}$ & WMS-R LM & + & $\mathrm{EM}$ & yes & (63) \\
\hline 40 & $\begin{array}{l}\text { Napryeyenko } \\
\text { O }\end{array}$ & 2007 & $\begin{array}{l}\text { Arzneimittelforschu } \\
\mathrm{ng}\end{array}$ & $A D$ or $\mathrm{VaD}$ & $>50$ & ginkgo biloba & $\begin{array}{l}\text { daily: } 2 \times 120 \mathrm{mg} \mathrm{EGb} \\
761\end{array}$ & unclear & 22 weeks & 395 & SKT test battery & + & WM & $\begin{array}{l}\text { no (no raw } \\
\text { measures } \\
\text { available) }\end{array}$ & (64) \\
\hline 41 & Nasab NM & 2012 & J Pak Med Assoc & $\begin{array}{l}\text { mild-moderate } \\
\mathrm{AD}\end{array}$ & $50-75$ & ginkgo biloba & $120 \mathrm{mg}$ ginkgo & $\begin{array}{l}4,5 \mathrm{mg} \\
\text { rivastigmine } \\
\text { (cholinesterase } \\
\text { inhibitor) }\end{array}$ & 24 weeks & $\begin{array}{l}56(51 \\
\text { completed) }\end{array}$ & $\begin{array}{l}7 \text { minutes test, } \\
\text { MMSE }\end{array}$ & - & WM & yes & (65) \\
\hline
\end{tabular}


TABLE 1 | Continued

\begin{tabular}{|c|c|c|c|c|c|c|c|c|c|c|c|c|c|c|c|}
\hline $\begin{array}{l}\text { Study } \\
\text { no.\# }\end{array}$ & First author & $\begin{array}{l}\text { Publication } \\
\text { year }\end{array}$ & Journal & Population & $\begin{array}{l}\text { Age } \\
\text { range } \\
\text { (years) }\end{array}$ & $\begin{array}{l}\text { Name of the } \\
\text { examined } \\
\text { extract, } \\
\text { polyphenol } \\
\text { or sublass } \\
\text { (referred to } \\
\text { as main or } \\
\text { effective } \\
\text { ingredient } \\
\text { by the } \\
\text { authors) }\end{array}$ & $\begin{array}{l}\text { Intervention, amount } \\
\text { of polyphenols }\end{array}$ & Control & $\begin{array}{l}\text { Duration of } \\
\text { intervention/ } \\
\text { control } \\
\text { period }\end{array}$ & $\mathbf{n}$ & $\begin{array}{l}\text { Cognitive tests } \\
\text { related to working } \\
\text { or episodic } \\
\text { memory } \\
\text { performance }\end{array}$ & $\begin{array}{l}\text { Any } \\
\text { significant } \\
\text { outcome }{ }^{\circ} \\
\text { none, + yes } \\
\text { positive; - } \\
\text { yes } \\
\text { negative) }\end{array}$ & $\begin{array}{l}\text { Significant } \\
\text { outcomes } \\
\text { in } \\
\text { working } \\
\text { (WM) or } \\
\text { episodic } \\
\text { memory } \\
\text { (EM), or } \\
\text { both }\end{array}$ & $\begin{array}{l}\text { included in } \\
\text { metaanalysis }\end{array}$ & Reference \\
\hline 42 & Nilsson A & 2017 & PLoS One & healthy adults & $50-70$ & anthocyanins & $\begin{array}{l}\text { 3×200ml berrymix } \\
(1325 \mathrm{mg} /=811 \mathrm{mg} / \mathrm{d})\end{array}$ & $\begin{array}{l}\text { placebo } \\
\text { (matched to } \\
\text { ingredients, } \\
\mathrm{pH} \\
\text { etc) less fibre } \\
\text { in the control } \\
\text { drink }\end{array}$ & 5 weeks & $\begin{array}{l}46(40 \\
\text { analysed, } \\
\text { crossover) }\end{array}$ & $\begin{array}{l}\text { dedicated sentence } \\
\text { list - WM test }\end{array}$ & $\circ$ & & yes & (66) \\
\hline 43 & Ochiai R & 2019 & J Alzheimers Dis & $\mathrm{MCl}$ & $60-84$ & $\begin{array}{l}\text { chlorogenic } \\
\text { acid }\end{array}$ & $2 \times 553.6 \mathrm{mg}$ CGA/d & $\begin{array}{l}\text { placebo } \\
\text { (identical wo } \\
\text { CDGs)! }\end{array}$ & 12 weeks & $\begin{array}{l}34(28 \\
\text { analysed), } \\
\text { crossover }\end{array}$ & ADAScog, TMT & + & WM & yes & (67) \\
\hline 44 & Pase MP & 2013 & $\begin{array}{l}\text { J } \\
\text { Psychopharmacol }\end{array}$ & healthy adults & $40-65$ & $\begin{array}{l}\text { cocoa } \\
\text { flavanols }\end{array}$ & $\begin{array}{l}500 \mathrm{mg}+250 \mathrm{mg} \\
\text { cocoa flavanols }\end{array}$ & $\begin{array}{l}\text { protein, sugar, } \\
\text { fat- } \\
\text { matched; all } \\
\text { had caffeine }\end{array}$ & 30 days & $\begin{array}{l}87(77 \\
\text { analysed) }\end{array}$ & CDR battery & 。 & & yes & (68) \\
\hline 45 & Pipingas A & 2008 & Phytother Res & $\begin{array}{l}\text { cognitively intact } \\
\text { adults }\end{array}$ & $50-65$ & flavonoids & $\begin{array}{l}960 \mathrm{mg} \text { of Enzogenol@ } \\
\text { and } 120 \mathrm{mg} \text { of vitamin } \\
\mathrm{C} \text {. Enzogenol@ is an } \\
\text { aqueous extract from } \\
\text { the bark of New } \\
\text { Zealand grown Pinus } \\
\text { radiata trees containing } \\
\text { approximately } 80 \% \\
\text { total proanthocyanins } \\
\text { and other } \\
\text { water-soluble } \\
\text { flavonoids, flavonoid- } \\
\text { conjugates and } \\
\text { phenolic acids. Rich in } \\
\text { proanthocyanins and } \\
\text { contain a range of } \\
\text { flavonoids including } \\
\text { catechin, epicatechin, } \\
\text { quercetin, } \\
\text { dihydroquercetin, } \\
\text { taxifolin and phenolic } \\
\text { acids }\end{array}$ & vitamin c only & 5 weeks & 42 & $\begin{array}{l}\text { computer-based } \\
\text { cognitive tasks for } \\
\text { spatial working } \\
\text { memory }\end{array}$ & + & WM & yes & (69) \\
\hline 46 & $\begin{array}{l}\text { Rainey- } \\
\text { Smith } \\
\text { SR }\end{array}$ & 2016 & BrJ Nutr & healthy adults & $40-90$ & curcuminoids & $\begin{array}{l}1500 \mathrm{mg} / \mathrm{d} \text { total }(1 \\
\times 500 \mathrm{mg} \\
\text { BCM-95®CG } \\
\text { (BiocurcumaxTM) } \\
\text { capsule three times a } \\
\text { day): }\end{array}$ & $\begin{array}{l}\text { placebo } \\
\text { (unclear) }\end{array}$ & 12 months & 160 & $\begin{array}{l}\text { sixteen-item self- } \\
\text { report Prospective } \\
\text { and Retrospective } \\
\text { Memory } \\
\text { Questionnaire; Rey } \\
\text { Auditory Verbal } \\
\text { Learning Test; } \\
\text { Subtests of } \\
\text { Cogstate battery }\end{array}$ & 。 & & yes & (70) \\
\hline
\end{tabular}


TABLE 1 | Continued

\begin{tabular}{|c|c|c|c|c|c|c|c|c|c|c|c|c|c|c|c|}
\hline $\begin{array}{l}\text { Study } \\
\text { no.\# }\end{array}$ & First author & $\begin{array}{l}\text { Publication } \\
\text { year }\end{array}$ & Journal & Population & $\begin{array}{l}\text { Age } \\
\text { range } \\
\text { (years) }\end{array}$ & $\begin{array}{l}\text { Name of the } \\
\text { examined } \\
\text { extract, } \\
\text { polyphenol } \\
\text { or sublass } \\
\text { (referred to } \\
\text { as main or } \\
\text { effective } \\
\text { ingredient } \\
\text { by the } \\
\text { authors) }\end{array}$ & $\begin{array}{l}\text { Intervention, amount } \\
\text { of polyphenols }\end{array}$ & Control & $\begin{array}{l}\text { Duration of } \\
\text { intervention/ } \\
\text { control } \\
\text { period }\end{array}$ & $n$ & $\begin{array}{l}\text { Cognitive tests } \\
\text { related to working } \\
\text { or episodic } \\
\text { memory } \\
\text { performance }\end{array}$ & $\begin{array}{l}\text { Any } \\
\text { significant } \\
\text { outcome }\left(^{\circ}\right. \\
\text { none, + yes } \\
\text { positive; - } \\
\text { yes } \\
\text { negative) }\end{array}$ & $\begin{array}{l}\text { Significant } \\
\text { outcomes } \\
\text { in } \\
\text { working } \\
\text { (WM) or } \\
\text { episodic } \\
\text { memory } \\
\text { (EM), or } \\
\text { both }\end{array}$ & $\begin{array}{l}\text { tincluded in } \\
\text { metaanalysis }\end{array}$ & Reference \\
\hline 47 & Ryan J & 2008 & $\begin{array}{l}J \\
\text { Psychopharmacol }\end{array}$ & healthy adults & $60-85$ & flavonoids & $3 \times 50 \mathrm{mg}$ PYC & $\begin{array}{l}\text { placebo } \\
\text { (same size } \\
\text { and } \\
\text { appearance), } \\
\text { unknown }\end{array}$ & 3 months & $\begin{array}{l}120(101 \\
\text { completed) }\end{array}$ & CDR battery & + & WM & yes & (71) \\
\hline 48 & Santos RF & 2003 & Pharmacopsychiatryh & Mhealthy men & $60-70$ & ginkgo biloba & $\begin{array}{l}\text { 80mg extract } \\
\text { (24\%flavonoids) }\end{array}$ & $\begin{array}{l}\text { placebo } \\
\text { (same size } \\
\text { and } \\
\text { appearance), } \\
\text { unknown }\end{array}$ & 8 months & $\begin{array}{l}48 \text { (all } \\
\text { completers) }\end{array}$ & test battery & + & WM & yes & (72) \\
\hline 49 & $\begin{array}{l}\text { Schneider } \\
\text { LS }\end{array}$ & 2005 & $\begin{array}{l}\text { Curr Alzheimer } \\
\text { Res }\end{array}$ & $\begin{array}{l}\text { mild-moderate } \\
\mathrm{AD}\end{array}$ & $>60$ & ginkgo biloba & $\begin{array}{l}1 \times 120 \mathrm{mg} \text { ginkgo or } \\
2 \times 120 \mathrm{mg}(240 \mathrm{mg}) \\
\text { ginkgo }\end{array}$ & placebo & 26 weeks & $\begin{array}{l}513(410 \\
\text { completed) }\end{array}$ & ADAS Cog & 。 & & $\begin{array}{l}\text { no (no raw } \\
\text { measures } \\
\text { available) }\end{array}$ & (73) \\
\hline 50 & $\begin{array}{l}\text { Schneider } \\
\text { LS }\end{array}$ & 2019 & Menopause & $\begin{array}{l}\text { perimenopausal } \\
\text { women }\end{array}$ & $45-60$ & isoflavones & $\begin{array}{l}50 \mathrm{mg}+100 \mathrm{mg} / \mathrm{d} \\
\text { phytoserm } \\
\text { (=isoflavones) }\end{array}$ & $\begin{array}{l}\text { placebo } \\
\text { (same size } \\
\text { and } \\
\text { appearance), } \\
\text { unknown }\end{array}$ & 12 weeks & $\begin{array}{l}71(66 \\
\text { completed) }\end{array}$ & composite measures & 。 & & $\begin{array}{l}\text { no (no raw } \\
\text { measures } \\
\text { available) }\end{array}$ & (74) \\
\hline 51 & Siddarth $\mathrm{P}$ & 2020 & Am J Clin Nutr & normal or $\mathrm{MCl}$ & $50-75$ & anthocyanins & $\begin{array}{l}236,5 \mathrm{ml} \\
\text { pomegranate/d } \\
\text { (368mg punicalagins, } \\
\text { ca } 100 \text { anthocyanins, } \\
\text { etc }\end{array}$ & $\begin{array}{l}\text { placebo } \\
\text { (same taste, } \\
\text { sugar etc) }\end{array}$ & 12 months & $\begin{array}{l}261(200 \\
\text { completed) }\end{array}$ & $\begin{array}{l}\text { Brief Visuospatial } \\
\text { Memory } \\
\text { Test-Revised } \\
\text { (BVMT-R) and } \\
\text { Buschke Selective } \\
\text { Reminding Test } \\
\text { (SRT) }\end{array}$ & + & EM & yes & (75) \\
\hline 52 & Small BJ & 2014 & Rejuvenation Res & $\begin{array}{l}\text { cognitively intact } \\
\text { adults }\end{array}$ & $65-85$ & anthocyanins & $\begin{array}{l}900 \mathrm{mg} \text { NT-020 } \\
\text { PlusBiovin / day } \\
\text { (containing green tea } \\
\text { extract ( } 95 \% \\
\text { polyphenols), VitaBlue } \\
\text { (40\% polyphenols, } \\
12.5 \% \text { anthocyanins } \\
\text { from blueberries), } \\
\text { grape polyphenols } \\
\text { (including } 5 \% \\
\text { resveratrol), carnosine, } \\
\text { vitamin D3 (2000 } \\
\text { UI/serving) and Biotin } \\
40 \mathrm{mg} \text {. }\end{array}$ & $\begin{array}{l}\text { placebo } \\
\text { (unclear) }\end{array}$ & 2 months & 113 & $\begin{array}{l}\text { Auditory Verbal } \\
\text { Learning Test (AVLT; } \\
\text { immediate and } \\
\text { delayed recall) voor } \\
\text { episodic memory; }\end{array}$ & o & & yes & (76) \\
\hline 53 & Snitz BE & 2009 & JAMA & $\begin{array}{l}\text { healthy elderly, } \\
\mathrm{MCl}\end{array}$ & $72-96$ & ginkgo biloba & $2 \times 120 \mathrm{mg}$ ginkgo & $\begin{array}{l}\text { placebo } \\
\text { (unknown) }\end{array}$ & 6,1 years & $\begin{array}{l}3072(1545 \\
+1542 \\
\text { completed) }\end{array}$ & $\begin{array}{l}\text { Stroop, TMT and } \\
\text { other }\end{array}$ & 0 & & yes & (77) \\
\hline 54 & Solomon PR & 2002 & JAMA & healthy adults & $60-82$ & ginkgo biloba & $3 \times 40 \mathrm{mg}$ ginkgo/day & $\begin{array}{l}\text { gelatine } \\
\text { capsules }\end{array}$ & 6 weeks & $\begin{array}{l}230(203 \\
\text { completed) }\end{array}$ & $\begin{array}{l}\text { Digit span, Stroop } \\
\text { and other }\end{array}$ & $\circ$ & & yes & (78) \\
\hline
\end{tabular}


TABLE 1 | Continued

\begin{tabular}{|c|c|c|c|c|c|c|c|c|c|c|c|c|c|c|c|}
\hline $\begin{array}{l}\text { Study } \\
\text { no.\# }\end{array}$ & First author & $\begin{array}{l}\text { Publication } \\
\text { year }\end{array}$ & Journal & Population & $\begin{array}{l}\text { Age } \\
\text { range } \\
\text { (years) }\end{array}$ & $\begin{array}{l}\text { Name of the } \\
\text { examined } \\
\text { extract, } \\
\text { polyphenol } \\
\text { or sublass } \\
\text { (referred to } \\
\text { as main or } \\
\text { effective } \\
\text { ingredient } \\
\text { by the } \\
\text { authors) }\end{array}$ & $\begin{array}{l}\text { Intervention, amount } \\
\text { of polyphenols }\end{array}$ & Control & $\begin{array}{l}\text { Duration of } \\
\text { intervention/ } \\
\text { control } \\
\text { period }\end{array}$ & $\mathbf{n}$ & $\begin{array}{l}\text { Cognitive tests } \\
\text { related to working } \\
\text { or episodic } \\
\text { memory } \\
\text { performance }\end{array}$ & $\begin{array}{l}\text { Any } \\
\text { significant } \\
\text { outcome }{ }^{\circ} \\
\text { none, + yes } \\
\text { positive; - } \\
\text { yes } \\
\text { negative) }\end{array}$ & $\begin{array}{l}\text { Significant } \\
\text { outcomes } \\
\text { in } \\
\text { working } \\
\text { (WM) or } \\
\text { episodic } \\
\text { memory } \\
\text { (EM), or } \\
\text { both }\end{array}$ & $\begin{array}{l}t \text { included in } \\
\text { metaanalysis }\end{array}$ & Reference \\
\hline 55 & $\begin{array}{l}\text { Suominen } \\
\text { MH }\end{array}$ & 2020 & Exp Gerontol & healthy adults & $65-75$ & $\begin{array}{l}\text { cocoa } \\
\text { flavanols }\end{array}$ & $\begin{array}{l}50 \mathrm{~g} \text { dark chocolate } \\
\text { (410mg flavanols/day) }\end{array}$ & $\begin{array}{l}50 \mathrm{~g} \text { dark } \\
\text { chocolate } \\
\text { (86mg } \\
\text { flavanols/ day) }\end{array}$ & 8 weeks & $\begin{array}{l}104 \text { (100 } \\
\text { completed) }\end{array}$ & TMT & 。 & & yes & (79) \\
\hline 56 & $\begin{array}{l}\text { Thaung Zaw } \\
\text { JJ }\end{array}$ & 2021 & Clin Nutr & $\begin{array}{l}\text { postmenopausal } \\
\text { women }\end{array}$ & 45-85 & resveratrol & $75 \mathrm{mg}$ trans-resveratrol & $\begin{array}{l}\text { several inert } \\
\text { excipients }\end{array}$ & 14 weeks & $\begin{array}{l}146 \text { ( } 110 \\
\text { analysed, } \\
\text { cross-over) }\end{array}$ & $\begin{array}{l}\text { TMT, list sorting, } \\
\text { wordlist, picture } \\
\text { memory }\end{array}$ & + & WM, EM & yes & (80) \\
\hline 57 & $\begin{array}{l}\text { van Dongen } \\
\mathrm{M}\end{array}$ & 2003 & J Clin Epidemiol & $\begin{array}{l}\text { mild-moderate } \\
\mathrm{AD} / \\
\mathrm{VaD} \text { or } \\
\text { age-associated } \\
\text { cognitive } \\
\text { impairment }\end{array}$ & $>50$ & ginkgo biloba & $\begin{array}{l}240 \mathrm{mg} / \text { day ginkgo } \\
\text { biloba special extract } \\
\text { (high dose) or } 160 \\
\text { (usual dose) } \mathrm{mg} / \text { day }\end{array}$ & $\begin{array}{l}\text { placebo } \\
\text { (unclear) }\end{array}$ & 24 weeks & 214 & $\begin{array}{l}\text { NAl-ZN-G; } \\
\text { self-perceived health } \\
\text { and memory status }\end{array}$ & 。 & & yes & (81) \\
\hline 58 & Whyte AR & 2018 & Nutrients & healthy adults & $65-80$ & anthocyanins & $\begin{array}{l}450 \mathrm{mg} / \text { day blueberry } \\
\text { powder (35mg } \\
\text { polyphenols, } 1.35 \mathrm{mg} \\
\text { anthocyanins)or } \\
900 \mathrm{mg} \text { blueberry } \\
\text { powder }(70 \mathrm{mg} \\
\text { polyphenols, } 2.7 \mathrm{mg} \\
\text { anthocyanins) or } \\
100 \mathrm{mg} \text { blueberry } \\
\text { extract (50mg } \\
\text { polyphenols, } 7 \mathrm{mg} \\
\text { anthocyanins) }\end{array}$ & maltodextrin & 6 months & $\begin{array}{l}122(112 \\
\text { completed) }\end{array}$ & $\begin{array}{l}\text { AVLT, object } \\
\text { recognition, serial } \\
\text { substractions, } \\
\text { Stroop }\end{array}$ & $\circ$ & & $\begin{array}{l}\text { no (no raw } \\
\text { measures } \\
\text { available) }\end{array}$ & (82) \\
\hline 59 & $\begin{array}{l}\text { Wightman } \\
\text { EL }\end{array}$ & 2018 & Nutrients & healthy adults & $50-70$ & flavonoids & $\begin{array}{l}475 \mathrm{mg} \text { or } 950 \mathrm{mg} / \text { day } \\
\text { Sideritis scardica } \\
\text { (Greek Mountain Tea) } \\
\text { or } 240 \mathrm{mg} \text { ginkgo/day }\end{array}$ & maltodextrin & 28 days & $\begin{array}{l}155 \text { ( } 140 \\
\text { completed) }\end{array}$ & $\begin{array}{l}\text { own tests, global } \\
\text { scores available }\end{array}$ & + & WM & yes & (83) \\
\hline 60 & Witte AV & 2014 & J Neurosci & $\begin{array}{l}\text { healthy } \\
\text { overweight } \\
\text { adults }\end{array}$ & $50-80$ & resveratrol & $\begin{array}{l}200 \mathrm{mg} / \mathrm{day} \text { of } \\
\text { resveratrol and } 320 \mathrm{mg} \\
\text { of quercetin }\end{array}$ & $\begin{array}{l}\text { placebo (corn } \\
\text { oil) }\end{array}$ & 26 weeks & 46 & $\begin{array}{l}\text { Auditory Verbal } \\
\text { Learning Test (AVLT) }\end{array}$ & + & EM & yes & (84) \\
\hline 61 & Wong RH & 2013 & J Hypertens & $\begin{array}{l}\text { obese healthy } \\
\text { men and } \\
\text { postmenopausal } \\
\text { women }\end{array}$ & $40-75$ & resveratrol & $75 \mathrm{mg}$ trans-resveratrol & $\begin{array}{l}\text { placebo } \\
\text { (identical } \\
\text { W/o } \\
\text { resveratrol) }\end{array}$ & 6 weeks & $\begin{array}{l}28 \\
\text { (cross-over) }\end{array}$ & Stroop & $\circ$ & & yes & (85) \\
\hline 62 & Woo J & 2003 & Menopause & $\begin{array}{l}\text { postmenopausal } \\
\text { women }\end{array}$ & 50-65 & isoflavones & $100 \mathrm{mg}$ isoflavones & $\begin{array}{l}\text { hormone } \\
\text { replacement } \\
\text { (estrogen/ } \\
\text { progesterone) } \\
\text { OR no } \\
\text { treatment }\end{array}$ & 3 months & $\begin{array}{l}127 \\
\text { (completers) }\end{array}$ & $\begin{array}{l}\text { TMT, word list } \\
\text { learning }\end{array}$ & $\circ$ & & yes & (86) \\
\hline
\end{tabular}


TABLE 1 | Continued

\begin{tabular}{|c|c|c|c|c|c|c|c|c|c|c|c|c|c|c|c|}
\hline $\begin{array}{l}\text { Study } \\
\text { no.\# }\end{array}$ & First author & $\begin{array}{l}\text { Publication } \\
\text { year }\end{array}$ & Journal & Population & $\begin{array}{l}\text { Age } \\
\text { range } \\
\text { (years) }\end{array}$ & $\begin{array}{l}\text { Name of the } \\
\text { examined } \\
\text { extract, } \\
\text { polyphenol } \\
\text { or sublass } \\
\text { (referred to } \\
\text { as main or } \\
\text { effective } \\
\text { ingredient } \\
\text { by the } \\
\text { authors) }\end{array}$ & $\begin{array}{l}\text { Intervention, amount } \\
\text { of polyphenols }\end{array}$ & Control & $\begin{array}{l}\text { Duration of } \\
\text { intervention/ } \\
\text { control } \\
\text { period }\end{array}$ & $\mathrm{n}$ & $\begin{array}{l}\text { Cognitive tests } \\
\text { related to working } \\
\text { or episodic } \\
\text { memory } \\
\text { performance }\end{array}$ & $\begin{array}{l}\text { Any } \\
\text { significant } \\
\text { outcome ( } \\
\text { none, + yes } \\
\text { positive; - } \\
\text { yes } \\
\text { negative) }\end{array}$ & $\begin{array}{l}\text { Significant } \\
\text { outcomes } \\
\text { in } \\
\text { working } \\
\text { (WM) or } \\
\text { episodic } \\
\text { memory } \\
\text { (EM), or } \\
\text { both }\end{array}$ & $\begin{array}{l}\text { t included in } \\
\text { metaanalysis }\end{array}$ & Reference \\
\hline 63 & Yakoot M & 2013 & Clin Interv Aging & $\mathrm{MCl}$ & $50-80$ & ginkgo biloba & $\begin{array}{l}\text { G. biloba leaf extract } \\
120 \mathrm{mg} \text { (standardized } \\
\text { to contain } 24 \% \\
\text { flavonoid glycosides } \\
\text { and } 6 \% \text { terpenoids) } \\
\text { and } 150 \mathrm{mg} \text { of P. } \\
\text { ginseng alcohol root } \\
\text { extract (containing } \\
40 \%-80 \% \\
\text { ginsenosides). }\end{array}$ & $\begin{array}{l}750 \mathrm{mg} \text { of } \\
\text { natural } \\
\text { lyophilized } \\
\text { royal } \\
\text { jelly } \\
\text { (standardized } \\
\text { to at least 6\% } \\
\text { of } \\
\text { 10-hydroxy- } \\
\text { 2decenoic } \\
\text { acid) }\end{array}$ & 4 weeks & $\begin{array}{l}66 \text { (60 } \\
\text { completed) }\end{array}$ & MMSE & + & WM & $\begin{array}{l}\text { no (unspecific } \\
\text { outcome) }\end{array}$ & (87) \\
\hline 64 & Yancheva S & 2009 & Aging Ment Health & $\begin{array}{l}\text { probable AD w } \\
\text { neuropsychiatric } \\
\text { symptoms }\end{array}$ & $>50$ & ginkgo biloba & $\begin{array}{l}2 \times 120 \mathrm{mg}(240 \mathrm{mg} / \text { day } \\
\text { ginkgo) or combined } \\
\text { with donezepil } \\
5-10 \mathrm{mg} / \text { day }\end{array}$ & $\begin{array}{l}5-10 \mathrm{mg} \\
\text { donezepil }\end{array}$ & 22 weeks & $\begin{array}{l}96(88 \\
\text { completed) }\end{array}$ & SKT & 。 & & $\begin{array}{l}\text { no (no raw } \\
\text { measures } \\
\text { available) }\end{array}$ & (88) \\
\hline 65 & You YX & 2021 & Nutrients & $\mathrm{MCl}$ & $60-75$ & flavonoids & $\begin{array}{l}\text { 2×250mg CC } \\
+250 \text { maltodextrin }\end{array}$ & $\begin{array}{l}2 \times 500 \mathrm{mg} \\
\text { maltodextrin }\end{array}$ & 12 weeks & $\begin{array}{l}48 \text { (47 } \\
\text { completed) }\end{array}$ & digit span, AVLT & + & WM, EM & yes & (89) \\
\hline 66 & Zhang SJ & 2012 & $\begin{array}{l}\text { Asian Pac J Trop } \\
\text { Med }\end{array}$ & $\begin{array}{l}\text { cerebral } \\
\text { infarction } \\
\text { patients/vascular } \\
\text { cognitive } \\
\text { impairment }\end{array}$ & $60-75$ & ginkgo biloba & $\begin{array}{l}3 \times 75 \mathrm{mg} \text { aspirin/day }+ \\
3 \times 40 \mathrm{mg} \text { ginkgo }\end{array}$ & $\begin{array}{l}\text { 3x75mg } \\
\text { aspirin/day }\end{array}$ & 3 months & 80 & MOCA & + & WM, EM & yes & (90) \\
\hline
\end{tabular}


To analyse overall effect sizes of the included studies and a potential positivity bias in the publication records, we compared effect sizes of WM and EM outcomes, respectively, with forest and funnel plots in the software JASP developed and distributed by JASP Team (91), JASP (Version 0.14.1) [Computer software]. Inference of a random effects meta-analysis per memory category (EM and WM, respectively) was computed with the restricted maximum likelihood methods by pooling all polyphenol interventions and subsequently with sub-analyses in subgroups.

\section{RESULTS}

\section{Study Characteristics}

The included 66 studies had study durations varying between 4 weeks and 2.5 years and sample sizes between 42 and 350 participants. The investigated polyphenol extracts were administered as tablets, capsules, powder, chocolate bar or drink and comprised a large variety of polyphenol molecules. According to Truzzi et al. (92), polyphenols can be classified in four subclasses according to the number of inert phenolic rings, i.e., flavonoids, phenolic acids, stilbenes and lignans; others that do not fall into those categories are polyphenols such as e.g., curcuminoids. To compare the studies included in this review, we decided to group studies according to the examined extract, polyphenol substance or polyphenol subclass that the authors described as main or most effective ingredient. This resulted in the following subcategories: Ginkgo biloba leaf extract (26 studies), soy isoflavones extracts (11 studies), anthocyanins (eight studies, e.g., blueberry extract, pomegranate, cherry juice, etc.), cocoa flavanols (four studies), flavanoid extracts (five studies, e.g., Sideritis scardica, Pinus radiata bark, etc.), chlorogenic acids (two studies), curcuminoids (four studies), and resveratrol (five studies). Note that all subcategories except chlorogenic acids, curcuminoids, and resveratrol belong to the same subclass of flavonoids (92), and that most extracts contained multiple polyphenols, leading to a certain overlap between study subgroups.

The majority of studies reported to use a placebo tablet, capsule, powder or drink as a control condition for supplementary polyphenols. These placebos comprised for example the intervention formula but only with negligible or no amounts of polyphenols, for example in tablets, capsules or drinks. Some studies incorporated isocaloric maltodextrin or cow milk protein (i.e., for soy isoflavone) as placebo, or studied the effects of polyphenols alone, or added to a certain treatment, e.g., fish oil or vitamins or aspirin, compared to those treatments alone, sometimes including a third arm without any treatment/placebo. Other studies, in some cases including 3-4 study arms, tested effects of polyphenols vs. medications, such as rivastigmin or donezepil in the case of cognitive decline, or hormone replacement therapy in the case of peri/postmenopausal women, or in comparison to lower doses of the polyphenol, for example flavanols. For more information about the study characteristics, (see Table 1).

\section{Adverse Events}

Most, but not all studies reported on adverse events. Some studies did this extensively, others scantly reported on adverse events. Overall, polyphenols were well-tolerated. Most often, when adverse events were reported, the number and type of adverse events did not differ significantly from the placebo group. One study on Ginkgo biloba effects reported the occurrence of stroke in one participant, which could not equivocally related to the treatment (38). Recurring complaints in the reviewed studies were gastrointestinal complaints.

\section{Reported Effects of Polyphenols on Memory}

Overall, in this systematic review, 32 out of 66 studies (48.5\%) found at least one significant improvement on any of the memory outcomes measured, while 31 (47\%) did not report significant performance, and $3(4.5 \%)$ did report decreases in memory in at least one measure (Table 1). However, these 66 studies analyzed a multitude of different outcome measures and different polyphenol substances or mixtures. All studies included at least one WM test outcomes, and the majority did also report EM outcomes. However, studies including dementia patients most often included only global assessments with relatively coarse measures of memory performance, such as the ADAS-Cog or SKIT, due to the severity of disease. This somewhat limits the interpretability of possible polyphenol effects on episodic memory in patients suffering more severe cognitive decline.

Tests assessing WM usually ask participants to remember information for a short amount of time and to manipulate this information. Examples of tests that measure WM are the digit span task, where participants have to repeat a series of numbers in the same or reversed order, or the serial subtraction task, where participants are assigned to keep on subtracting the number three or seven from a random number between 800 and $999(18,53)$. Another frequent test was the trail making test (TMT) B, which gives a working memory estimate of mental flexibility. Assessing EM typically entails the visual or verbal presentation of information to a participant and later measures whether this information is remembered (22). An example of an EM test is the Rey Auditory Verbal Learning Test (RAVLT), where participants hear a list of 15 words spoken out loud and are asked to recall the words immediately and after a delay and are asked what words they recognized from a 30-words list comprising old, new and distractor words (53). Forty-five studies included healthy older adults, with some focusing on overweight/obese participants and 12 included only peri/postmenopausal women, one only men. Eleven studies included subjective (SMI) or minor neurocognitive impairment (MCI), while another 14 studies included dementia of the Alzheimer's type (AD) or vascular dementia (VaD), or both. Depending on population, most studies had a relatively broad age range older than 40,45 , or 50 , some focused on $65-80$ years old only. 


\section{Effects of Polyphenols on Working and Episdoic Memory (WM/EM) Ginkgo biloba}

Almost half of the studies included in the review, i.e., 26, investigated the effects of Ginkgo biloba, in both healthy and disease samples, two with ginseng added to the treatment. Ginkgo biloba leaf extract is comprised of different polyphenols but particularly_similar to soy or certain other plant extracts-rich in flavonoids. The duration of treatment lasted from 4 weeks to up to 3.5 years. Fourteen of those studies reported at least one significant improvement, 11 no changes and two a worsening of functions. Notably, two of the longest studies over 3.5 and 6.5 years, respectively, reported diverging results: the first in 202 completers found a significant beneficial effect of $120 \mathrm{mg} /$ day ginkgo tablets compared to placebo tablets in the ADAS-cog test and the GEFRI test $(p=0.04)$ in dementia patients (56), while the second longer one in a very large group ( $n=3,072$ completers) and after a doubled dosage (240 mg Ginkgo biloba) could not detect significant differences in healthy older or MCI patients (77). Due to the large sample size and long duration, the latter study is of particular interest, as well as the first one, however we could not include it in the meta-analysis based on missing specific memory outcomes (56). Two shorter studies in mild to moderate $\mathrm{AD}$ with doubled dosage over 6 months compared to placebo in around 400 participants also reported benefits $(46,64)$. However, other large trials with 410/214 completers reported no significant differences for improvements after 6 months in the ADAS cog test (73) (Table 1). Some studies also included subjective measures of memory performance, which is by some regarded as biomarker of later cognitive function ("subjective memory impairment"), however a less objective measure of current memory performance and thus not further considered in this review. Notably, two studies reported a worsening of function compared to control condition, one in healthy adults after $160 \mathrm{mg} /$ day in 78 completers (however for EM outcome), and another one in patients with mild to moderate $\mathrm{AD}$ when comparing $120 \mathrm{mg} /$ day ginkgo to $4.5 \mathrm{mg} /$ day rivastigmin treatment (a cholinesterase inhibitor).

\section{Soy Isoflavones}

Isoflavones show similarities with steroidal estrogens and are suggested to counteract menopause-related estrogen deficiency possibly causing cognitive decline $(24,47)$. Soy isoflavones were examined in nine studies in peri- or postmenopausal women, one study included also men and another one AD patients. Overall, most studies $(72 \%)$ could not detect any significant effects of isoflavones (mostly from soy) in dosages between 35 and 160 mg/day over 3-12 months, including a relatively large study with 175 women over the course of 1 year (53). One study in 79 women over three groups reported a worsening of a verbal working memory outcome after 16 weeks of soy isoflavone intake as soymilk, compared to cow milk or isoflavone supplement intervention (40). A cross-over study of 6 months isoflavone tablets compared to placebo $(n=76)$, however, as well as larger $(n=313)$, longer term intervention of soy isoflavones against milk protein-based placebo over 2.5 years, showed improvements in a working memory outcome [TMTB (34)] and in episodic memory (24), respectively. It could be speculated that isoflavones are effective in earlier age only because of their possible action as estrogen-related effects. Some studies indeed showed that effects are mainly found several years before menopause (i.e., perimenopause), when the ovaries start to produce less estrogen, or in young postmenopausal women $(93,94)$. However, two studies in perimenopausal women included in this review do not support this claim (41).

\section{Berry, Cherry, Grape and Tea Extracts, Curcuminoids, and Related Supplements}

Berry, cherry, grape, and green tea extracts are among other polyphenols rich in anthocyanins, evaluated in nine studies. Other extracts included cocoa flavanols (studied in four studies), flavonones (one study), curcuminoids (four studies), and rosmarinic acids (one study). Overall, study results were mixed with nine studies reporting no significant effects and nine studies reporting significant outcomes for WM or EM tasks. One study reported improvements in spatial and WM, but not EM, after intake of rosmarinic acid, containing spearmint extract for 90 days compared to identical placebo supplement ( $0 \mathrm{mg}$ spearmint), after three months in a group of 87 healthy older adults $(45,69)$. Considering anthocyanins, for example administered as grape juice or blueberry extracts, most studies including healthy participants could not detect significant changes over 5 weeks to 6 months (four studies). For example, one study performed in 113 healthy elderly did not find any significant improvements on WM or EM outcome measures between the experimental and control group after 2 months (76). Study dosages were partly unclear, as for example, Whyte et al. (82) administered between 35 and $70 \mathrm{mg}$ polyphenols per day and Small et al. (76) reported administering $900 \mathrm{mg}$ of the supplement NT-020 per day, but it was unclear what the amount of which polyphenol was per dosage. In a relatively large sample $(n=122)$ of elderly with subjective self-reported memory complaints (82), the group receiving low dose polyphenols per day, including $7 \mathrm{mg}$ anthocyanin per day, showed a significant improvement compared to placebo on one EM outcome measure after 3 months of supplementation, but not after 6 months. No significant improvements were found on the other five outcome measures of the verbal learning task or on a visual EM task.

In a population of 49 older adults with mild-to-moderate dementia and $138 \mathrm{mg}$ anthocyanins per day intervention, however, Kent et al. (13) found significant improvements on all three measures of EM after 3 months, with moderate to large effect sizes (13). Bensalem et al. (31) reported dosing $258 \mathrm{mg}$ flavonoids per day and showed a significant improvement in one of the three EM outcome measures after 6 months in a sample of 215 healthy elderly (31), and a secondary analysis revealed that polyphenol supplementation significantly improved all three measures of EM in a subgroup with the highest cognitive decline.

The influence of flavanols derived from cocoa was measured in both cognitively intact elderly and elderly with mild neurocognitive disorder $(37,59)$. In two studies, results showed that participants drinking high and medium amounts of flavanols performed significantly better on a WM task than participants 
drinking low amounts of flavanols. In Mastroiacovo et al. (59), the high and medium flavanol groups reduced the time to finish a WM task over 8 weeks with $17 \mathrm{~s}(21 \%)$ and $14 \mathrm{~s}(18 \%)$ respectively, compared to $1 \mathrm{~s}(1 \%)$ in the low flavanol group. However, two studies in 77-100 healthy adults could not detect significant effects after 4-8 weeks intervention with daily drinks or chocolate bars against a protein shake or a low-dose flavanol chocolate, respectively, as control $(68,79)$. A similar impression comes from four studies on curcuminoids, with two showing no significant results in 134-160 healthy olders after 16 weeks-12 months intervention $(55,70)$ and two studies from the same group in 60-79 participants reporting significant improvements in working memory measures after 4 and 12 weeks $(18,36)$. Here, it seems as if a shorter study duration (i.e., 4 weeks) more likely resulted in positive results, while an intervention period of 1 year did not lead to a difference in composite scores between the placebo and intervention group. On the other hand, the power of the studies with insignificant results was bigger, as the sample size were larger. The dosages of those studies are difficult to compare. Cox et al. (18) for example administered $80 \mathrm{mg}$ curcumin per day. However, Rainey-Smith et al. (70) reported administering $1,320 \mathrm{mg}$ curcuminoids but not mentioning the precise amount of other polyphenols.

In sum, the evidence for a beneficial effect on EM or WM on anthocyanins, flavanols and curcuminoids, is still limited, with a tendency toward more positive effects when cognitive decline is more severe based on two studies only. In line with this, though, the sample of Small et al. (76), showing no significant differences at all, was highly educated and therefore a ceiling effect of the memory task cannot be ruled out. Note also a lack of homogeneity with regard to dosages of different polyphenol subclasses, rendering a direct comparison of study results difficult: some of the flavanol studies for instance varied between 990 and $520 \mathrm{mg}$ flavanol per day, while in the isoflavone studies, lower but still varying doses of 35-160 mg of isoflavones were administered.

\section{Stilbenes}

Supplementary doses of isolated polyphenols were rare and often combined with others derivatives or substances, such as in studies examining resveratrol, a polyphenol of the stilbene subclass (five studies). These studies tested postmenopausal women and healthy older adults with again mixed results. For example, Evans et al. (39) administered $150 \mathrm{mg}$ resveratrol per day for over 14 weeks to 80 participants, while Huhn et al. (48) administered $200 \mathrm{mg}$ per day plus $320 \mathrm{mg}$ quercetin for over 26 weeks to 60 participants. Considering WM, although Huhn et al. (48) found no significant improvements, pattern recognition on a spatial WM-related test decreased in the placebo group and did not change in the resveratrol group. This result could suggest that resveratrol helps to preserve cognitive function, while memory otherwise subtly declines with aging. However, this effect was not seen in Evans et al. (39), where the performance in both the placebo and the resveratrol group increased with about the same amount. While quercetin was given to increase bioavailability of resveratrol, it cannot be excluded that a positive result related to the flavonoid-related benefits of quercetin, administered simultaneously in Huhn et al. (48). Considering EM, significant improvements were found in a sample of 46 healthy older adults and in a sample of 80 postmenopausal women $(39,84)$. Witte et al. (84) administered $200 \mathrm{mg}$ resveratrol and $320 \mathrm{mg}$ quercetin for 26 weeks. In both studies, the positive effect was only found on delayed recall and not on immediate recall of a verbal learning task. According to Evans et al. (39), the effect size was small. The above mentioned study by Huhn et al. (48) researching 60 healthy elderly could not confirm these findings, as no significant improvements in delayed recall, nor on three other outcome measures of a verbal learning task were found after 26 weeks (48). Again, it is imported to highlight that both Witte et al. (84) and Huhn et al. (48) also administered quercetin, to increase the bioavailability of resveratrol, so the results of these studies might be a combined effect of flavonoids and stilbenes.

Related, while one study did not report significant differences after resveratrol in obese older adults (85), others studied an overweight population and found significant improvement in EM performance after resveratrol administration (84). Here, the increased EM performance was associated with improvements in glucose metabolism (i.e., lower HbA1c levels). In addition, ameliorated memory performance was associated with increased functional connectivity between the hippocampus and the medial prefrontal cortex, suggesting a mechanistic link. In addition, a study with cocoa flavanol drink observed significant reductions in blood insulin after treatment, which also explained variance in the memory increases after treatment (55), again pointing to glucose metabolism as a potential mechanism. Positive verbal memory results for $75 \mathrm{mg} /$ day trans-resveratrol intake compared to placebo capsules were also found in postmenopausal women in a very recent study after 14 weeks each in a cross-over design, including 110 women (80).

\section{Evaluation of Effect Size}

Considering the difference between the reviewed studies, overall, results appeared to be independent of sample size and study duration. A substantially larger sample size, leading to a greater power to detect an effect that is present, did not always lead to significant results in some samples, similar to smaller samples, suggesting that the effect of polyphenols on memory depends on other factors.

Taken together, available effect sizes of WM outcomes after polyphenol intake interventions appeared in the majority either small or non-existent, with the exception of some studies in both directions and two studies reporting extremely large positive effects on immediate face recognition (24) and TMT-B (59) (Figure 2).

When pooled in a meta-analysis, polyphenols showed a small overall positive effect on working memory [Wald test, $b=0.26$ (95\% CI: 0.09; 0.43), $z=3.07, p=0.002]$. The between-study heterogeneity variance was estimated at $\tau^{2}=0.44$ (95\% CI: $0.32-$ 0.7 ), with an $I^{2}$ value of $93 \%$ (95\% CI: 91-96\%), indicating that statistical heterogeneity between studies was highly significantly large $\left[Q_{(66)}=710, p<0.001\right]$. The associated funnel plot indicated a positivity bias in the publication record, according to visual inspection and Egger's regression test $(p=0.017)$ and a trend in the rank correlation test (Kendall's $\tau=0.15, p=$ 
Ahles S 2020 WM (stroop)

Beck SM 2016 WM (delayed response, switch)

Beck SM 2016 WM (task-set switching)

Bensalem J 2019 WM (reverse spatial span)

Bensalem J 2019 WM (spatial span)

Burns NR 2006 WM (immediate recall)

Burns NR 2006 WM (processing speed)

Carlson JJ 2007 WM (visual-spatial working memory)

Casini ML 2006 WM (digit span bw)

Cieza A 2003 WM (connection trial)

Cieza A 2003 WM (stroop)

Cox KH 2015 WM (serial three subtraction)

Cox KHM 2020 WM (serial 7)

Cox KHM 2020 WM(spatial learning)

Desideri G 2012 WM (TMT B)

Evans HM 2017 WM (spatial span)

Evans HM 2017 WM (TMT-B)

Furlong ON 2020 WM (spatial span)

Gavrilova SI 2014 WM (TMT B)

Gleason CE 2015 WM (stroop)

Gleason CE 2015 WM (TMT B)

Hartley DE 2004 WM (DMTS, 12sec)

Henderson VW 2012 WM (CVLT, immediate)

Henderson VW 2012 WM (faces, immediate)

Herrlinger KA 2018 WM (verbal composite)

Ho SC 2007 WM (stroop)

Ho SC 2007 WM (visuospatial)

Huhn S 2018 WM (ModBent)

Huhn S 2018 WM (TMT B)

Jackson PA 2016 WM (RVIP accuracy)

Jackson PA 2016 WM (substraction 7)

Kaschel R 2011 WM (verbal)

Kean RJ 2015 WM (verbal pairs immediate)

Kean RJ 2015 WM (visuospatial)

Kent K 2017 WM (digit span)

Kent K 2017 WM (SOPT)

Kreijkamp-Kaspers S 2004 WM (digit span)

Kreijkamp-Kaspers S 2004 WM (TMT B)

Kritz-Silverstein D 2003 WM (TMT-B)

Li S 2019 WM (visuospatial)

Mastroiacovo D 2015 WM (TMT-B)

Mix JA 2002 WM (digit symbol)

Nasab NM 2012 WM (sevenmin test)

Nilsson A 2017 WM (sentences)

Ochiai R 2019 WM (TMT-B)

Pase MP 2013 WM (quality of working memory)

Pipingas A 2008 WM (visuospatial)

Rainey-Smith SR 2016 WM (verbal immediate)

Ryan J 2008 WM (spatial)

Ryan J 2008 WM (numeric)

Santos RF 2003 WM (corsiblock)

Santos RF 2003 WM (digit span)

Small BJ 2014 WM (digit span)

Small BJ 2014 WM (TMT-B)

Snitz BE 2009 WM (exec function, global)

Solomon PR 2002 WM (digit span)

Solomon PR 2002 WM (stroop)

Suominen MH 2020 WM (TMT B)

Thaung Zaw JJ 2021 WM (flexibility composite)

Thaung Zaw JJ 2021 WM (working memory composite)

van Dongen M 2003 WM (digit span)

Wightman EL 2018 WM (global). 1

Wightman EL 2018 WM (global).2

Wong RH 2013 WM (Stroop)

Woo J 2003 WM (TMTB)

Woo J 2003 WM (verbal learning)

You YX 2021 WM (digit span)

You YX 2021 WM (immediate vr)

Zhang SJ 2012 WM (trails)

RE Model

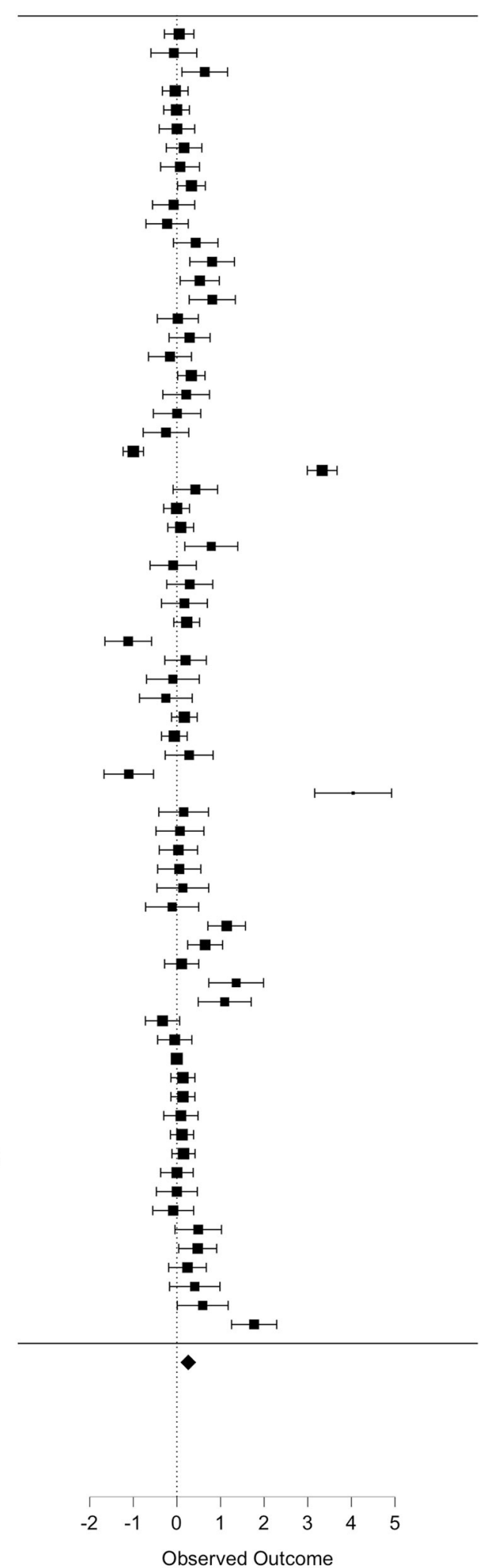

Observed Outcome
$0.05[-0.29,0.39]$

$-0.07[-0.60,0.45]$

$0.64[0.11,1.16]$

$-0.04[-0.33,0.25]$

$-0.01[-0.30,0.28]$

$0.00[-0.41,0.41]$

$0.16[-0.24,0.57]$

$0.07[-0.37,0.52]$

$0.33[0.01,0.65]$

$-0.08[-0.56,0.41]$

$-0.23[-0.71,0.26]$

$0.43[-0.08,0.94]$

$0.81[0.29,1.32]$

$0.52[0.07,0.97]$

$0.81[0.28,1.34]$

$0.02[-0.45,0.49]$

$0.29[-0.18,0.76]$

$-0.16[-0.65,0.33]$

$0.33[0.02,0.64]$

$0.21[-0.32,0.75]$

$0.00[-0.54,0.55]$

$-0.25[-0.77,0.27]$

$-1.00[-1.23,-0.77]$

$3.33[2.99,3.67]$

$0.42[-0.09,0.93]$

$-0.01[-0.30,0.29]$

$0.09[-0.21,0.38]$

$0.79[0.18,1.39]$

$-0.09[-0.62,0.44]$

$0.29[-0.23,0.82]$

$0.17[-0.35,0.70]$

$0.22[-0.07,0.52]$

$-1.12[-1.65,-0.58]$

$0.20[-0.28,0.67]$

$-0.09[-0.70,0.51]$

$0.17[-0.12,0.46]$

$-0.06[-0.35,0.23]$

$0.28[-0.27,0.83]$

$-1.10[-1.67,-0.54]$

$4.04[3.16,4.92]$

$0.15[-0.41,0.72]$

$0.07[-0.48,0.62]$

$0.03[-0.40,0.47]$

$0.05[-0.44,0.55]$

$0.14[-0.46,0.73]$

$-0.11[-0.72,0.50]$

$1.14[0.71,1.57]$

$0.65[0.25,1.05]$

$0.11[-0.28,0.50]$

$1.36[0.73,1.99]$

$1.09[0.49,1.70]$

$-0.33[-0.72,0.06]$

$-0.05[-0.44,0.34]$

$-0.00[-0.07,0.07]$

$0.14[-0.14,0.41]$

$0.14[-0.14,0.41]$

$0.09[-0.30,0.48]$

$0.12[-0.15,0.38]$

$0.15[-0.11,0.42]$

$0.00[-0.37,0.37]$

$-0.00[-0.47,0.47]$

$-0.09[-0.55,0.38]$

$0.49[-0.04,1.02]$

$0.48[0.04,0.91]$

$0.24[-0.19,0.67]$

$0.41[-0.17,0.99]$

$0.59[0.01,1.17]$

$1.77[1.25,2.29]$

$0.26[0.09,0.43]$

FIGURE 2 | Forest plot for observed effect sizes (d) and standard errors (SE) for available study results for WM. RE, regression estimate. 


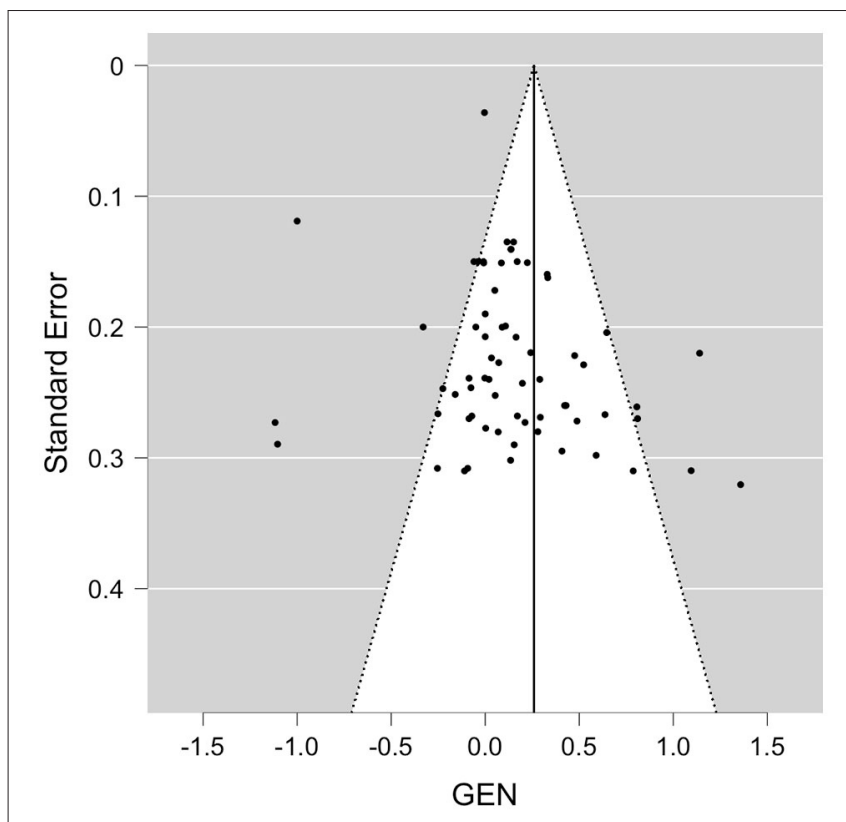

FIGURE 3 | Funnel plot for available study results for WM. GEN, general effect result per study.

0.065) (Figure 3). To further evaluate whether the two relatively large RCTs $(n=90-313)$ that added large positive effect sizes to the meta-analysis had considerable influence on the results, we re-run the meta-analysis excluding these studies $(24,59)$, however, while the pooled effect size became somewhat smaller, significance level remained unchanged $[b=0.29, z=3.6, p<$ 0.001 (95\% CI: 0.08-0.28)], with not much lowered betweenstudy heterogeneity $\left[I^{2}=77 \%, Q_{(65)}=205, p<0.001\right]$. Note that a positivity bias could no longer be observed (Rank test and Egger's test, $p>0.18$ ). When focusing on study outcomes that derived from comparisons of placebo formulas without polyphenols or no treatment as control condition (i.e., excluding study outcomes from comparison to lower dosages as control, or from polyphenols compared to fish oil, medications or to alternative extracts), overall results remained stable to the overall meta-analysis (59 outcomes, $b=0.26, p>0.001$, Rank test $p$ $=0.045)$.

Studies including mainly healthy samples showed similarly a significant effect size on average, yet the likelihood of a positivity bias in those studies was increased (majority of studies, 55 outcomes), $p<0.032$. In studies including mild cognitive impairment or dementia patients (14 outcomes), though, no positivity bias emerged, and effects were no longer significant $(b$ $=0.2, p=0.224)$. Duration of studies did not affect meta-analysis outcomes that much ( $<6$ months: $b=0.19, p=0.011 ; 6$ months or longer: $b=0.47, p=0.053)$, however Funnel asymmetry could not be excluded, and the majority of studies tested $<6$ months of intervention period. When restricting analyses for studies on ginkgo (23 outcomes, 17 studies, note also a large proportion of patient samples here), meta-analysis on effect sizes did not reach significance for a positive effect $(b=0.2, p=0.075)$ and a positivity bias based on Funnel plot asymmetry was no longer observed $(p>0.35)$. Similarly, meta-analysis for isoflavones (12 outcomes, eight studies; $b=0.3, p=0.28$, Funnel asymmetry, $p$ $>0.11$ ), anthocyanins (eight outcomes, five studies; $b=-0.06$, $p=0.38$, Funnel asymmetry $p>0.25$ ) and flavonoids (eight outcomes, five studies; $b=0.1 ; p=0.62$, asymmetry $p>0.64)$ did not show significant effects. However, studies examining other extracts containing resveratrol (seven outcomes, four studies; $b$ $=0.19, p=0.013$; asymmetry $p>0.22$ ) supported a significant positive average effect size. Note that sample size in the subgroupmeta analyses was considerably reduced. No subanalysis was computed in groups with $<5$ outcomes.

Evaluating EM, the included effect sizes indicated a small positive effect of polyphenol intake on EM outcomes on average (Figure 4). Accordingly, polyphenols showed a significant effect on episodic memory when pooled in a meta-analysis [Wald test, $b=0.24, z=2.88, p=0.004$ (95\% CI: 0.08-0.41)]. The between-study heterogeneity variance was moderate to large, estimated at $\tau^{2}=0.31$ (95\% CI: $0.21-0.54$ ), with an $I^{2}$ value of $91 \%$ (95\% CI: $88-95 \%)$, pointing toward a high statistical heterogeneity between studies $\left[Q_{(50)}=430, p<0.001\right]$. Notably, both the rank correlation test (Kendall's $\tau=0.34, p<0.001$ ) and the Egger's regression test $(p=0.01)$ indicated significant asymmetry of the associated funnel plot. The funnel plot did not indicate according to visual inspection more strongly positive effect sizes had been reported in larger studies than could have been expected (Figure 5). Three small to large RCTs with small to longest durations added large effect sizes of positive sign (delayed recognition of faces, verbal recall) to the meta-analysis. When excluding these study $(13,24,90)$, though, the pooled positive effect size became somewhat smaller yet remained statistically significant $[b=0.21, z=2.39, p=0.017$ (95\% CI: 0.02-0.18)], with a reduced between-study heterogeneity $\left(I^{2}=51 \%\right)$ yet still evidence for positivity bias (asymmetry, $p>0.021$ ). When focusing on study outcomes that derived from comparisons of placebo formulas without polyphenols or no treatment as control condition (i.e., excluding study outcomes from comparison to lower dosages as control, or from polyphenols compared to fish oil, medications or to alternative extracts), overall results remained stable to the overall meta-analysis (47 outcomes, $b=$ 0.23, $p=0.009$, Rank test $p<0.001$ ).

Note that a meta-analysis of those studies including mainly healthy samples did not reach statistical significance, yet the likelihood of a positivity bias in those studies was decreased majority of studies, 40 outcomes, $b=0.14, p=0.069$; Funnel asymmetry, $p>0.052$. In studies including mild cognitive impairment or dementia patients (14 outcomes), effects were again significant, with a smaller estimate $(b=0.64, p=$ 0.019 ) and again positivity bias (Funnel asymmetry, $p<0.032$ ). Duration of studies did not affect meta-analysis outcomes that much ( $<6$ months: $b=0.23, p=0.02 ; 6$ months or longer: $b$ $=0.25, p=0.097$ ), however Funnel asymmetry could not be excluded, and the majority of studies tested again $<6$ months of intervention period. When restricting analyses for studies on ginkgo (17 outcomes, 13 studies, note also a large proportion of patient samples here), meta-analysis on effect sizes did not reach significance for a positive effect $(b=0.22, p=0.19)$ and 
Beck SM 2016 EM (prospective memory)

Bensalem J 2019 EM (verbal recall)

Bensalem J 2019 EM (verbal recognition)

Burns NR 2006 EM (delayed recall)

Burns NR 2006 EM (long-term storage and retrieval)

Carlson JJ 2007 EM (wordlist)

Cieza A 2003 EM (wordlist)

Cox KHM 2020 EM(spatial memory)

Evans HM 2017 EM (verbal delayed)

Furlong ON 2020 EM (pattern recognition)

Gleason CE 2015 EM (delayed visual memory)

Gleason CE 2015 EM (verbal learning, free recall)

Hartley DE 2004 EM (composite)

Henderson VW 2012 EM (faces, delayed)

Henderson WW 2012 EM (CVLT, delayed)

Herrlinger KA 2018 EM (verbal composite)

Ho SC 2007 EM (verbal delayed)

Ho SC 2007 EM (visual delayed)

Kaschel R 2011 EM (delayed recall)

Kean RJ 2015 EM (verbal pairs delayed)

Kean RJ 2015 EM (verbal wordlist)

Kent K 2017 EM (verbal, 20 min)

Kent K 2017 EM (verbal)

Kreijkamp-Kaspers S 2004 EM (verbal delayed recall)

Kreijkamp-Kaspers S 2004 EM (visual)

Kritz-Silverstein D 2003 EM (verbal)

Li S 2019 EM (Moca delayed recall)

Mix JA 2002 EM (verball delayed recall)

Mix JA 2002 EM (visual delayed recognition)

Pase MP 2013 EM (composite)

Pipingas A 2008 EM (delayed recognition)

Rainey-Smith SR 2016 EM (verbal delayed recall)

Ryan J 2008 EM (verbal delayed wm)

Santos RF 2003 EM (verbal delayed)

Santos RF 2003 EM (visuospatial delayed recognition)

Siddarth P 2020 EM (delayed verbal recall)

Siddarth P 2020 EM (visuospatial delayed recall)

Small BJ 2014 EM (delayed recall)

Snitz BE 2009 EM (global memory)

Solomon PR 2002 EM (CVLT delayed)

Solomon PR 2002 EM (spatial)

Thaung Zaw JJ 2021 EM (episodic memory)

Thaung Zaw JJ 2021 EM (verbal memory)

Wightman EL 2018 EM (global).1

Wightman EL 2018 EM (global).2

Witte AV 2014 WM (delayed recall)

Witte AV 2014 WM (retention)

Woo J 2003 EM (delayed recall)

You YX 2021 EM (delayed vr)

You YX 2021 EM (verbal delayed)

Zhang SJ 2012 EM (verbal)

RE Model



Observed Outcome 


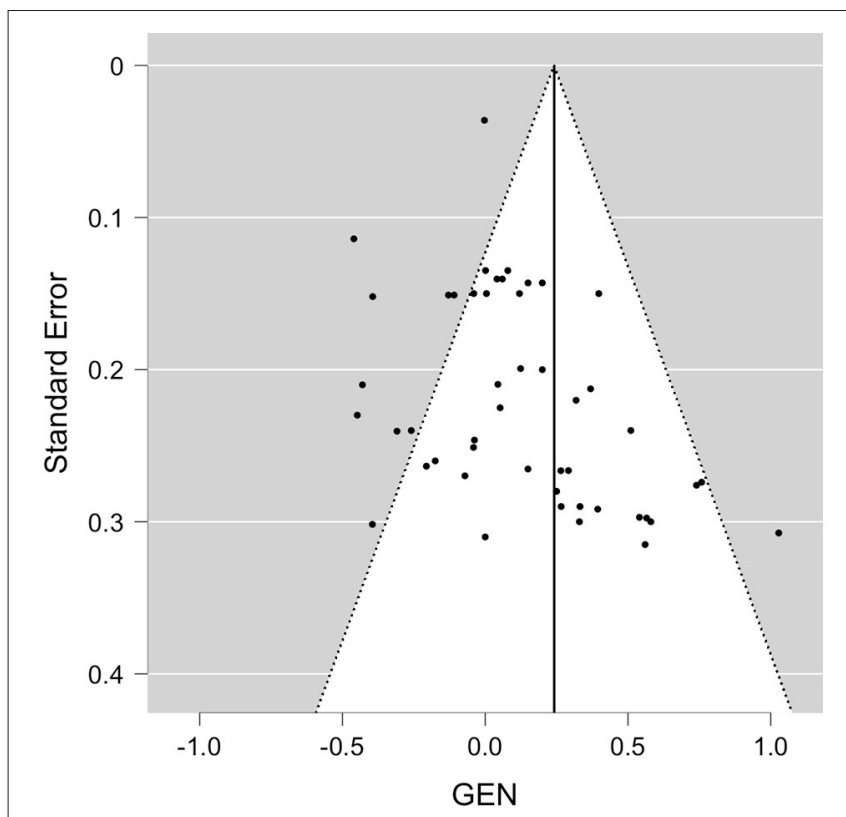

FIGURE 5 | Funnel plot for available study results for EM. GEN, general effect result per study.

a positivity bias based on Funnel plot asymmetry could still not be excluded (Rank test, $p=0.006$ ). Similarly, meta-analysis for isoflavones (11 outcomes, seven studies; $b=0.23 p=0.28$, Funnel asymmetry, $p>0.24$ ) did not show significant effects. In contrast, the meta-analysis for anthocyanins (seven outcomes, four studies; $b=0.45, p=0.05$, Funnel asymmetry Egger's test, $p=0.001$ ), for other extracts containing flavonoids (seven outcomes, five studies; $b=0.34 ; p=0.029$ Funnel asymmetry $p>0.3$ ) and trend-wise for resveratrol (five outcomes, three studies; $b=0.21 ; p=0.067$ asymmetry Egger's test, $p=0.024$ ) somewhat indicated a positive average effect size, yet both groups raised awareness with regard to positivity bias. Note that sample size in the subgroup meta-analyses was considerably reduced. No subanalysis was computed in groups with $<5$ outcomes.

\section{DISCUSSION}

Based on this systematic review of 66 reported RCTs testing the effects of polyphenols on memory, 33 studies found a significant improvement on at least one memory outcome measure after polyphenol administration, while 30 did not find any significant effects, and three reported a worsening compared to control condition. Reporting was based on a variety of WM and EM outcome measures, of which a selection only were reported as significantly improved after polyphenol consumption compared to placebo with, if reported, small to large effect sizes. Considering the available and calculated effect sizes from available outcomes of 49 studies representing core WM and EM measures, pooled meta-analyses supported a small positive effect on both WM and EM with a mean effect size of 0.26 and 0.24 , respectively. However, Funnel plot asymmetry tests detected a significant positivity bias for both WM and EM meta-analysis, questioning the validity of results. When excluding studies with very large positive effects, though, meta-analyses remained significant for a small effect. Our review further indicated a large heterogeneity between studies and outcomes studied, in terms of polyphenol formula and exact memory test used, study duration, sample size and characteristics, as well as considering statistical variance in effects sizes.

\section{Working and Episodic Memory}

The evaluated studies can be summarized to show a small positive effect on both WM and EM with similar estimates, however, note that this finding needs to be interpreted with caution due the indicated positivity bias in reporting of polyphenol studies. Still, it has been argued that polyphenols might improve brain function. For example, Brickman et al. (95) showed that specific parts of the hippocampus are activated after polyphenol consumption. When high cocoa-flavanol consumption was compared to low consumption, the middle part (i.e., the body) of the hippocampus was activated. The activation was also associated with higher memory performance. It should be noted though, that this study was performed in a small sample of 37 participants divided into two groups. The hippocampus is necessary for accessing short-term memory representations during WM tasks $(20,96)$, and hippocampal and parahippocampal areas, parts of the medial temporal lobe (MTL), are critically involved in EM for encoding and retrieval $(20,96)$, whereas MTL lesions do not lead to extreme WM performance degradation. Later studies however indicated the involvement of the MTL in WM tasks, especially when associations (e.g., location and color) need to be made (96-98).

Future studies involving neuroimaging techniques could help to further understand the neurobiological mechanisms underlying potential benefits of polyphenols on WM and EM.

\section{Possible Sources of Study Heterogeneity or Bias}

In this review, we did not observe strong systematic associations between memory improvement and sample size, study duration and mean age. Indeed, significant results were found in studies with long-term follow-up assessment after 2.5 years and in those with follow-up after 6 weeks. Additionally, bigger sample sizes, and thus greater power to detect an effect that is present did not result in more (in)significant findings. Also, the mean age of the samples did not systematically lead to significant or insignificant study results, however, some differences were observed regarding study population and polyphenol subclass.

\section{Polyphenol Subclasses and Dosage}

Overall, results of the polyphenol subclasses were mixed. The percentage of significant memory outcome measures for Ginkgo biloba, other polyphenol-rich extracts, flavonoids, phenolic acids, and stilbenes were relatively similarly distributed, while subgroup meta-analyses in polyphenol subclasses that could include five outcomes or more failed to show significant benefits for Ginkgo biloba, flavanol and soy isoflavones. Here, probability of positivity bias was markedly reduced, indicating a higher 
confidence in the results (though note the considerably smaller number of studies included in the sub-analyses). Some support for the expected significant positive effects were found for flavonoids, anthocyanins and resveratrol. In addition, within the polyphenol subclasses, efficiency was sometimes dependent on study dosage. For example, it can be speculated that the dosages in the studies administering Ginkgo biloba extract, namely between 38.4 and $57.6 \mathrm{mg}$ flavonoids per day, were too low to affect memory. The polyphenol dosages for Pinus radiata bark extract and spearmint extract were higher, namely $768 \mathrm{mg}$ flavonoids per day and $216 \mathrm{mg}$ polyphenols per day, respectively. Also, inter-individual differences in polyphenol absorption, metabolism, and excretion might be other reasons of diverging study results. For instance, Bensalem et al. (31) found that the group with the highest EM decline excreted significantly higher amounts of phenolic metabolites while this group consumed fewer polyphenols than the groups with better performing EM. Possibly, polyphenol supplementation in the group with the highest amount of cognitive decline compensates for the lower intake and higher excretion, leading to improved memory performances in people with more severe memory decline. However, as sample characteristics, exact polyphenol formulas and dosages varied considerably between studies, as well as metabolomics of polyphenols were seldomly reported, results are difficult to harmonize in this regard. A longitudinal observational study in $>2,500$ older adults also pointed toward the possibility that the effects of polyphenol subclass and molecule may be per se different but also with regard to their impact on cognitive domain: Here, higher intake of e.g., catechins and flavonols were related to both higher verbal memory performance after a period of more than 10 years, while this also correlated with lower performance in tasks on executive function (99). However note that as most extracts in the reviewed studies provided a mixture of polyphenolic molecules, a detailed cause-response investigation with regard to memory function seemed difficult. Future studies should incorporate blood-based biomarkers of individual polyphenol availability and metabolism, to further understand potential differential effects of polyphenol subclasses on cognitive performance.

\section{Impact of Age, Pathologies, and Gender}

Mild-to-moderate Alzheimer's disease patients were only studied in a small proportion of RCTs, and presenting on global cognitive test results such as the MMSE instead of WM/EM-focused subtests prevented from adding most of these to the meta-analyses. This underlines the difficulty to differentiate results between non-pathological and pathological aging populations. Considering results of subgroup metaanalyses, however, polyphenols seemed to exert positive effects in MCI and AD or vascular dementia patients on WM, but not significantly on EM, in contrast to meta-analyses in healthy groups. In addition, studies in older women only could not show significant improvements on average, yet, this might be also due to a lack of effects of isoflavonols, as this was the polyphenol used in most of these studies. Another interpretation is that both Ginkgo biloba and soy isoflavones had attracted probably the most interest in the last decades as a dietary supplement to combat cognitive decline, potentially due to certain hypes around alternative, nutrition-guided medicine approaches and to the estrogenic action attributed to isoflavones. Thus, these polyphenols were studied most extensively in the literature also with larger, longer-term and high-quality RCTs, leading to a more balanced reporting and resulting (partly) null findings, which would fit to some of the subgroup meta-analyses.

Still, differences in sample age, pathology and gender between studies could have contributed to mixed results. For example, positive effects of Pinus radiata bark and spearmint extracts were found in relatively young age, while no effects were seen in samples that were on average 10-25 years older. Moreover, on study that could not demonstrate any positive effect, meaning no objective or subjective effect, of a polyphenol-rich extract on memory, was performed in the oldest sample (81). This was a sample consisting of patients with dementia or ageassociated memory impairment. Consequently, it is possible that polyphenols are more effective in relatively young and unaffected samples, but less effective in older patient samples. In addition, menopause in women is associated with an increased risk for developing metabolic syndrome, known for disturbances in glucose metabolism (100). It has been hypothesized that the negative consequences of cardiovascular risk factors on memory performance, for example higher glucose levels, can be compensated by certain polyphenols such as resveratrol administration due to regulation of glucose metabolism and insulin sensitivity (101), rendering a possible efficacy of polyphenols in postmenopausal women likely. In a previous study in older adults, we found that resveratrol lowered glycated hemoglobin A1c in blood, a long-term marker of glucose, which was associated with improved functional connectivity of the hippocampus with the medial prefrontal cortex and eventually memory retention (84). Also, reductions in insulin after cocoa flavonols correlated with increases in memory performance, supporting a potential link between polyphenols and insulin sensitivity as beneficial mechanism (59).

However, samples were often not fully characterized with regard to cardiovascular and metabolic health as well as brain diseases, i.e., (clinical) blood and neuroimaging biomarker analysis has not always been performed. Future studies with larger, more diverse samples and sensitive monitoring of cardiovascular, metabolic and beginning brain pathologies are needed to better understand the impact of age, (beginning) pathologies, and gender on polyphenol action.

\section{Underlying Mechanisms}

Several mechanisms have been proposed that might underly the beneficial effects of polyphenols on brain aging. These include anti-oxidative and anti-inflammatory mechanisms and improvements in cardiovascular health such as lower blood pressure and better insulin sensitivity, which are all related to better brain structure and function. Pinus radiata bark extract, for example, has been discussed to inhibit oxidation, to reduce systolic blood pressure and to modify signaling in the brain due to the ability of polyphenol metabolites to cross the bloodbrain barrier (69). The reduction of oxidation byproducts in the hippocampus has been suggested as a possible mechanism 
of spearmint extract (45). Moreover, a recent meta-analysis of carotenoids, known to exert anti-oxidant properties in vitro and in vivo, provides evidence for a positive effect of carotenoids on improving cognitive performance in middle-aged and older adults, further supporting the hypothesis of a causal role of antioxidant actions in the beneficial effect of plant-derived nutrients on brain health (102). A reduction in pro-inflammatory markers has for instance been observed after anthocyaninrich supplements, thereby improving hippocampus-dependent memory performance. Kent et al. (13) did however not find altered levels of inflammatory markers in blood, although these alterations might have been undetectable due to disease progression. Yet, significantly lower systolic blood pressure was found by Kent et al. (13) and Whyte et al. (82) after polyphenol administration, which might be a consequence of reduced inflammatory markers and could relate to better memory functioning. For more details on suggested mechanistic pathways linking specific nutrients to cognitive function in aging please see other reviews [e.g., $(4,16,102-104)]$.

\section{Limitations}

Several limitations of this review should be considered. First, several studies could not be added to our meta-analysis due to lack of raw values, specific information or unplausibility in the given tables, limiting interpretability. Second, only one search base was included (PubMed), due to the limited access to Cochrane and EMBOS library, leading to a risk of omitting published study results. In addition, the results of the different types of polyphenols on WM and EM should be interpreted with care, since the number of studies per polyphenol subclass or extract was still relatively small. Thirdly, a wide variety of memory tests were used, and we cannot exclude that potential arbitrary differences in the classification to WM/EM category per author may have emerged (105). Only a few studies reported test validity and low construct validity might lead to drawing incorrect conclusions from the results. These factors might have contributed to the large statistical heterogeneity observed in the meta-analyses, and reported findings given in the systematic review may not withstand a tight control of type-1 error in the individual studies, in line with the often detected probable positivity bias. In addition, we did not evaluate included studies on study quality e.g., using established tools such as the GRADE or Cochrane risk of bias (106) due to the sheer number of studies screened, which could have revealed for example a lack of blinding or inadequate statistical reporting. However, all studies incorporated a randomized clinical trial design and a sample size of $n \geq 20$ per group, reducing the likelihood of extreme outliers. Also, doses of the different polyphenols were barely comparable and not harmonized at all. All studies reported dailyadministered amounts, but the effect of a specific dose of for example isoflavone is hard to compare with the effect of the same amount of flavanol, and compliance measures often relied on self-report or capsule count once at the end of studies.

In general, effect sizes in nutrition sciences and lifestyle interventions are expected to be rather small due to confounding factors in a free-living population. In contrast to other fields, lifestyle interventions have a long tradition of being preregistered, e.g., on ClinicalTrials.gov and nowadays also on osf.io, which enables to restrict the number of post-hoc statistical testing and the possibility to report null or negative findings. Indeed, effect sizes in pre-registered studies were shown to be smaller in pre-registered studies compared to non-pre-registered studies (107). Therefore, the herein presented results of a significant effect for EM and WM are likely to be expected and indeed representative of the field.

Future studies should harmonize control conditions and use memory tests with high construct validity and focus on the quality of the methods. Methodological quality can be increased by for example concealment of allocation, using an intention-to-treat analysis, and measuring compliance as well as implementing memory tests that are insensitive to ceiling or test-retest effects, such as the computerized administration of the Mnemonic Similarity Task (108). Moreover, future studies should include larger samples and longer follow-up to increase power. Yet, the sample sizes and durations of the RCTs included in this review did not seem to affect the results. By including biological parameters, such as urine or blood samples, and functional and structural brain measures using e.g., magnetic resonance imaging, more insights on the mechanisms of polyphenols in improving memory might be gained. Performing longer longitudinal studies in the future could provide insight into whether the consumption of polyphenols decreases progression rates of patients with mild neurocognitive disorder to dementia.

\section{CONCLUSION}

Based on reviewing 66 short- to longer-duration daily polyphenol intervention RCTs with small to large sample sizes, a beneficial effect of polyphenols on WM and EM in middle-aged to older adults may be considered small on average, according to qualitative review and a pooled meta-analysis of all available outcomes of 49 studies. The reported outcome measures largely varied and some studies of longer duration and larger sample sizes did not report any significant memory improvement after polyphenol administration. We also noted strong evidence for reporting bias and the statistical heterogeneity was considerably large between studies. Thus interpretation warrants caution and needs to be confirmed by further research. Future studies are encouraged to harmonize polyphenol formulas and doses as well as neuropsychological test methodology, and to increase sample sizes and follow-up periods. Overall, dietary supplementation studies investigating diet-effects on memory of high quality do exist, however, suffer from known limitations in the field and the problem to investigate rather small expected effects. Future studies should aim to address these challenges through rigorously implementing the advantages of open science, including data and code sharing, transparent reporting of neuropsychological methods and null/negative findings, and detailed pre-registration of RCTs, including a detailed statistical analysis plan, to increase reliability and to enable further metaanalyses and replication. 


\section{DATA AVAILABILITY STATEMENT}

The original contributions presented in the study are included in the article as meta-data. Further inquiries related to effect sizes per study or other queries can be directed to the corresponding author.

\section{AUTHOR CONTRIBUTIONS}

$\mathrm{KV}$, EM, and AVW: conceptualization and data analysis. KV and AVW: conducted literature

\section{REFERENCES}

1. United Nations, Department of Economic and Social Affairs. World Population Prospects 2019. (2019). Available online at: http://www.ncbi.nlm. nih.gov/pubmed/12283219 (accessed December 16, 2021).

2. Sarubbo F, Esteban S, Miralles A, Moranta D. Effects of resveratrol and other polyphenols on Sirt1: relevance to brain function during aging. Curr Neuropharmacol. (2017) 16:126-36. doi: 10.2174/1570159X15666170703113212

3. Rossini PM, Rossi S, Babiloni C, Polich J. Clinical neurophysiology of aging brain: from normal aging to neurodegeneration. Progr Neurobiol. (2007) 83:375-400. doi: 10.1016/j.pneurobio.2007.07.010

4. Sarubbo F, Moranta D, Pani G. Dietary polyphenols and neurogenesis: molecular interactions and implication for brain ageing and cognition. Neurosci Biobehav Rev. (2018) 90:45670. doi: 10.1016/j.neubiorev.2018.05.011

5. Alzheimer's Disease International. World Alzheimer Report 2015. The Global Impact of Dementia: An Analysis of Prevalence, Incidence, Cost and Trends. (2015).

6. Deary IJ, Corley J, Gow AJ, Harris SE, Houlihan LM, Marioni RE, et al. Age-associated cognitive decline. Br Med Bullet. (2009) 92:13552. doi: 10.1093/bmb/ldp033

7. Pusswald G, Tropper E, Kryspin-Exner I, Moser D, Klug S, Auff E, et al. Health-related quality of life in patients with subjective cognitive decline and mild cognitive impairment and its relation to activities of daily living. J Alzheimer's Dis. (2015) 47:479-86. doi: 10.3233/JAD-150284

8. Arvanitakis Z, Shah RC, Bennett DA. Diagnosis and management of dementia: review. J Am Med Assoc. (2019) 322:158999. doi: 10.1001/jama.2019.4782

9. Sanford AM. Mild cognitive impairment. Clin Geriatr Med. (2017) 33:32537. doi: 10.1016/j.cger.2017.02.005

10. Miquel S, Champ C, Day J, Aarts E, Bahr BA, Bakker M, et al. Poor cognitive ageing: vulnerabilities, mechanisms and the impact of nutritional interventions. Ageing Res Rev. (2018) 42:40-55. doi: 10.1016/j.arr.2017.12.004

11. Román GC, Jackson RE, Gadhia R, Román AN, Reis J. Mediterranean diet: the role of long-chain $\omega$-3 fatty acids in fish; polyphenols in fruits, vegetables, cereals, coffee, tea, cacao and wine; probiotics and vitamins in prevention of stroke, age-related cognitive decline, and Alzheimer disease. Revue Neurol. (2019) 175:724-41. doi: 10.1016/j.neurol.2019.08.005

12. Castelli V, Grassi D, Bocale R, D’Angelo M, Antonosante A, Cimini A, et al. Diet and brain health: which role for polyphenols? Curr Pharm Des. (2017) 24:227-38. doi: 10.2174/1381612824666171213100449

13. Kent K, Charlton K, Roodenrys S, Batterham M, Potter J, Traynor V, et al. Consumption of anthocyanin-rich cherry juice for 12 weeks improves memory and cognition in older adults with mild-to-moderate dementia. Eur J Nutr. (2017) 56:333-41. doi: 10.1007/s00394-015-1083-y

14. Bakoyiannis I, Daskalopoulou A, Pergialiotis V, Perrea D. Phytochemicals and cognitive health: are flavonoids doing the trick? Biomed Pharmacother. (2019) 109:1488-97. doi: 10.1016/j.biopha.2018.10.086

15. Bensalem J, Dal-Pan A, Gillard E, Calon F, Pallet V. Protective effects of berry polyphenols against age-related cognitive impairment. Nutr Aging. (2016) 3:89-106. doi: 10.3233/NUA-150051 search. KV: first draft. EM and AVW: visualization. EM, AVW, and AK: review and correction. All authors contributed to the article and approved the submitted manuscript.

\section{FUNDING}

This work was supported by grants of the German Research Foundation No. WI 3342/3-1 and 209933838 CRC105203 A1 (AVW) and by the German Federal Environmental Foundation (EM).

16. Huhn S, Kharabian Masouleh S, Stumvoll M, Villringer A, Witte AV. Components of a Mediterranean diet and their impact on cognitive functions in aging. Front Aging Neurosci. (2015) 7:132. doi: 10.3389/fnagi.2015. 00132

17. Spieth PM, Kubasch AS, Penzlin AI, Illigens BMW, Barlinn K, Siepmann T. Randomized Controlled Trials - A Matter of Design. Neuropsychiatric Disease and Treatment. Macclesfield: Dove Medical Press Ltd. (2016).

18. Cox KHM, Pipingas A, Scholey AB. Investigation of the effects of solid lipid curcumin on cognition and mood in a healthy older population. $J$ Psychopharmacol. (2015) 29:642-51. doi: 10.1177/0269881114552744

19. Verlinden VJA, Van Der Geest JN, De Bruijn RFAG, Hofman A, Koudstaal PJ, Ikram MA. Trajectories of decline in cognition and daily functioning in preclinical dementia. Alzheimer's Dement. (2016) 12:14453. doi: 10.1016/j.jalz.2015.08.001

20. Cabeza R, Dolcos F, Graham R, Nyberg L. Similarities and differences in the neural correlates of episodic memory retrieval and working memory. Neuroimage. (2002) 16:317-30. doi: 10.1006/nimg.2002.1063

21. Al-Hassan S, Duell N. The effect of working memory on stroop performance. In: Conference Abstract: 2nd International Conference on Educational Neuroscience (Abu Dhabi). (2017).

22. Luo L, Craik FIM. Aging and memory: a cognitive approach. Can J Psychiatr. (2008) 53:346-53. doi: 10.1177/070674370805300603

23. Dickerson BC, Eichenbaum H. The episodic memory system: neurocircuitry and disorders. Neuropsychopharmacology. (2010) 35:86-104. doi: 10.1038/npp.2009.126

24. Henderson VW, St John JA, Hodis HN, Kono N, McCleary CA, Franke $\mathrm{AA}$, et al. Long-term soy isoflavone supplementation and cognition in women: a randomized, controlled trial. Neurology. (2012) 78:18418. doi: 10.1212/WNL.0b013e318258f822

25. Ritchie K, Carrière I, Su L, O’Brien JT, Lovestone S, Wells K, Ritchie CW. The midlife cognitive profiles of adults at high risk of late-onset Alzheimer's disease: The PREVENT study. Alzheimers Dement. (2017) 13:1089-97. doi: 10.1016/j.jalz.2017.02.008

26. Park DC, Reuter-Lorenz P. The adaptive brain: aging and neurocognitive scaffolding. Annu Rev Psychol. (2009) 60:17396. doi: 10.1146/annurev.psych.59.103006.093656

27. Peters R. Ageing and the brain. Postgrad Med J. (2006) 82:848. doi: 10.1136/pgmj.2005.036665

28. Ahles S, Stevens YR, Joris PJ, Vauzour D, Adam J, de Groot E, et al. The effect of long-term aronia melanocarpa extract supplementation on cognitive performance, mood, and vascular function: a randomized controlled trial in healthy, middle-aged individuals. Nutrients. (2020) 12:258. doi: 10.3390/nu12082475

29. Basaria S, Wisniewski A, Dupree K, Bruno T, Song MY, Yao F, et al. Effect of high-dose isoflavones on cognition, quality of life, androgens, and lipoprotein in post-menopausal women. J Endocrinol Invest. (2009) 32:150-5. doi: 10.1007/BF03345705

30. Beck SM, Ruge H, Schindler C, Burkart M, Miller R, Kirschbaum C, et al. Effects of Ginkgo biloba extract EGb 761(R) on cognitive control functions, mental activity of the prefrontal cortex and stress reactivity in elderly adults with subjective memory impairment - a randomized doubleblind placebo-controlled trial. Hum Psychopharmacol. (2016) 31:227-42. doi: 10.1002/hup.2534 
31. Bensalem J, Dudonné S, Etchamendy N, Pellay H, Amadieu C, Gaudout D, et al. Polyphenols from grape and blueberry improve episodic memory in healthy elderly with lower level of memory performance: a bicentric doubleblind, randomized, placebo-controlled clinical study. J Gerontol Ser A Biol Sci Med Sci. (2019) 74:996-1007. doi: 10.1093/gerona/gly166

32. Burns NR, Bryan J, Nettelbeck T. Ginkgo biloba: no robust effect on cognitive abilities or mood in healthy young or older adults. Hum Psychopharmacol. (2006) 21:27-37. doi: 10.1002/hup.739

33. Carlson JJ, Farquhar JW, DiNucci E, Ausserer L, Zehnder J, Miller D, et al. Safety and efficacy of a ginkgo biloba-containing dietary supplement on cognitive function, quality of life, and platelet function in healthy, cognitively intact older adults. J Am Diet Assoc. (2007) 107:422-2. doi: $10.1016 /$ j.jada.2006.12.011

34. Casini ML, Marelli G, Papaleo E, Ferrari A, D’Ambrosio F, Unfer V. Psychological assessment of the effects of treatment with phytoestrogens on postmenopausal women: a randomized, doubleblind, crossover, placebo-controlled study. Fertil Steril. (2006) 85:972-8. doi: 10.1016/j.fertnstert.2005.09.048

35. Cieza A, Maier P, Pöppel E. Effects of Ginkgo biloba on mental functioning in healthy volunteers. Arch Med Res. (2003) 34:373-81. doi: 10.1016/j.arcmed.2003.05.001

36. Cox KHM, White DJ, Pipingas A, Poorun K, Scholey A. Further evidence of benefits to mood and working memory from lipidated curcumin in healthy older people: a 12-week, double-blind, placebo-controlled, partial replication study. Nutrients. (2020) 12:1678. doi: 10.3390/nu12061678

37. Desideri G, Kwik-Uribe C, Grassi D, Necozione S, Ghiadoni L, Mastroiacovo $\mathrm{D}$, et al. Benefits in cognitive function, blood pressure, and insulin resistance through cocoa flavanol consumption in elderly subjects with mild cognitive impairment the cocoa, cognition, and aging (CoCoA) study. Hypertension. (2012) 60:794. doi: 10.1161/HYPERTENSIONAHA.112.193060

38. Dodge HH, Zitzelberger T, Oken BS, Howieson D, Kaye J. A randomized placebo-controlled trial of Ginkgo biloba for the prevention of cognitive decline. Neurology. (2008) 70:180917. doi: 10.1212/01.wnl.0000303814.13509.db

39. Evans HM, Howe PRC, Wong RHX. Effects of resveratrol on cognitive performance, mood and cerebrovascular function in post-menopausal women; a 14-week randomised placebo-controlled intervention trial. Nutrients. (2017) 9:27. doi: 10.3390/nu9010027

40. Fournier LR, Ryan Borchers TA, Robison LM, Wiediger M, Park JS, Chew $\mathrm{BP}$, et al. The effects of soy milk and isoflavone supplements on cognitive performance in healthy, postmenopausal women. J Nutr Health Aging. (2007) 11:155-64.

41. Furlong ON, Parr HJ, Hodge SJ, Slevin MM, Simpson EE, McSorley EM, et al. Consumption of a soy drink has no effect on cognitive function but may alleviate vasomotor symptoms in post-menopausal women; a randomised trial. Eur J Nutr. (2020) 59:755-66. doi: 10.1007/s00394-01901942-5

42. Gavrilova SI, Preuss UW, Wong JW, Hoerr R, Kaschel R, Bachinskaya $\mathrm{N}$, et al. Efficacy and safety of Ginkgo biloba extract EGb 761 in mild cognitive impairment with neuropsychiatric symptoms: a randomized, placebo-controlled, double-blind, multi-center trial. Int J Geriatr Psychiatry. (2014) 29:1087-95. doi: 10.1002/gps.4103

43. Gleason CE, Fischer BL, Dowling NM, Setchell KD, Atwood CS, Carlsson $\mathrm{CM}$, et al. Cognitive effects of soy isoflavones in patients with Alzheimer's disease. J Alzheimer's Dis. (2015) 47:1009-19. doi: 10.3233/JAD-142958

44. Hartley DE, Elsabagh S, File SE. Gincosan (a combination of Ginkgo biloba and Panax ginseng): the effects on mood and cognition of 6 and 12 weeks' treatment in post-menopausal women. Nutr Neurosci. (2004) 7:325-33. doi: 10.1080/10284150400015557

45. Herrlinger KA, Nieman KM, Sanoshy KD, Fonseca BA, Lasrado JA, Schild $\mathrm{AL}$, et al. Spearmint extract improves working memory in men and women with age-associated memory impairment. J Altern Complement Med. (2018) 24:37-47. doi: 10.1089/acm.2016.0379

46. Herrschaft H, Nacu A, Likhachev S, Sholomov I, Hoerr R, Schlaefke S. Ginkgo biloba extract EGb 761(R) in dementia with neuropsychiatric features: a randomised, placebo-controlled trial to confirm the efficacy and safety of a daily dose of $240 \mathrm{mg}$. J Psychiatr Res. (2012) 46:71623. doi: 10.1016/j.jpsychires.2012.03.003
47. Ho SC, Chan ASY, Ho YP, So EKF, Sham A, Zee B, et al. Effects of soy isoflavone supplementation on cognitive function in Chinese postmenopausal women: a double-blind, randomized, controlled trial. Menopause. (2007) 14:489-99. doi: 10.1097/gme.0b013e31802c4f4f

48. Huhn S, Beyer F, Zhang R, Lampe L, Grothe J, Kratzsch J, et al. Effects of resveratrol on memory performance, hippocampus connectivity and microstructure in older adults - a randomized controlled trial. Neuroimage. (2018) 174:177-90. doi: 10.1016/j.neuroimage.2018.03.023

49. Jackson PA, Forster JS, Bell JG, Dick JR, Younger I, Kennedy DO. DHA supplementation alone or in combination with other nutrients does not modulate cerebral hemodynamics or cognitive function in healthy older adults. Nutrients. (2016) 8.

50. Kanowski S, Herrmann WM, Stephan K, Wierich W, Horr R. Proof of efficacy of the ginkgo biloba special extract EGb 761 in outpatients suffering from mild to moderate primary degenerative dementia of the Alzheimer type or multi-infarct dementia. Pharmacopsychiatry. (1996) 29:47-56. doi: 10.1055/s-2007-979544

51. Kaschel R. Specific memory effects of Ginkgo biloba extract EGb 761 in middle-aged healthy volunteers. Phytomedicine. (2011) 18:1202-7 doi: 10.1016/j.phymed.2011.06.021

52. Kean RJ, Lamport DJ, Dodd GF, Freeman JE, Williams CM, Ellis JA, et al. Chronic consumption of flavanone-rich orange juice is associated with cognitive benefits: an 8-wk, randomized, double-blind, placebocontrolled trial in healthy older adults. Am J Clin Nutr. (2015) 101:506-14. doi: 10.3945/ajcn.114.088518

53. Kreijkamp-Kaspers S, Kok L, Grobbee DE, de Haan EHF, Aleman A, Lampe JW, et al. Effect of soy protein containing isoflavones on cognitive function, bone mineral density, and plasma lipids in postmenopausal women - a randomized controlled trial. J Am Med Assoc. (2004) 292:6574. doi: 10.1001/jama.292.1.65

54. Kritz-Silverstein D, Von Muhlen D, Barrett-Connor E, Bressel MAB Isoflavones and cognitive function in older women: the soy and postmenopausal health in aging (SOPHIA) study. Menopause. (2003) 10:196-202. doi: 10.1097/00042192-200310030-00004

55. Kuszewski JC, Howe PRC, Wong RHX. Evaluation of cognitive performance following fish-oil and curcumin supplementation in middle-aged and older adults with overweight or obesity. J Nutr. (2020) 150:31909. doi: 10.1093/jn/nxaa299

56. Le Bars PL, Katz MM, Berman N, Itil TM, Freedman AM, Schatzberg AF. A placebo-controlled, double-blind, randomized trial of an extract of Ginkgo biloba for dementia. North American EGb Study Group. J Am Med Assoc. (1997) 278:1327-32. doi: 10.1001/jama.1997.03550160047037

57. Lewis JE, Melillo AB, Tiozzo E, Chen L, Leonard S, Howell M, et al. A doubleblind, randomized clinical trial of dietary supplementation on cognitive and immune functioning in healthy older adults. Bmc. (2014).

58. Li S, Cao GY, Deng QW, Zhu D, Yan FL. Effect of Pushen capsule for treating vascular mild cognitive impairment: a pilot observational study. J Int Med Res. (2019) 47:5483-96.

59. Mastroiacovo D, Kwik-Uribe C, Grassi D, Necozione S, Raffaele A, Pistacchio $\mathrm{L}$, et al. Cocoa flavanol consumption improves cognitive function, blood pressure control, and metabolic profile in elderly subjects: the Cocoa, Cognition, and Aging (CoCoA) study-a randomized controlled trial. Am J Clin Nutr. (2015) 101:538-48. doi: 10.3945/ajcn.114.092189

60. Mazza M, Capuano A, Bria P, Mazza S. Ginkgo biloba and donepezil: a comparison in the treatment of Alzheimer's dementia in a randomized placebo-controlled double-blind study. Eur J Neurol. (2006) 13:981-5. doi: 10.1111/j.1468-1331.2006.01409.x

61. McCarney R, Fisher P, Iliffe S, van Haselen R, Griffin M, van der Meulen J, et al. Ginkgo biloba for mild to moderate dementia in a community setting: a pragmatic, randomised, parallel-group, double-blind, placebo-controlled trial. Int J Geriatr Psychiatry. (2008) 23:1222-30. doi: 10.1002/gps.2055

62. McNamara RK, Kalt W, Shidler MD, McDonald J, Summerc SS, Stein AL, et al. Cognitive response to fish oil, blueberry, and combined supplementation in older adults with subjective cognitive impairment. Neurobiol Aging. (2018) 64:147-56. doi: 10.1016/j.neurobiolaging.2017.12.003

63. Mix JA, Crews WD. An examination of the efficacy of Ginkgo biloba extract EGb 761 on the neuropsychologic functioning of cognitively 
intact older adults. J Altern Complement Med. (2000) 6:219-29. doi: $10.1089 / \mathrm{acm} .2000 .6 .219$

64. Napryeyenko O, Borzenko I, Group G-NS. Ginkgo biloba special extract in dementia with neuropsychiatric features. A randomised, placebo-controlled, double-blind clinical trial. Arzneimittelforschung. (2007) 57:4-11. doi: 10.1055/s-0031-1296579

65. Nasab NM, Bahrammi MA, Nikpour MRA, Rahim F, Naghibi SN. Efficacy of rivastigmine in comparison to ginkgo for treating Alzheimer's dementia. JPMA. (2012) 62:677-80.

66. Nilsson A, Salo I, Plaza M, Bjorck I. Effects of a mixed berry beverage on cognitive functions and cardiometabolic risk markers; A randomized cross-over study in healthy older adults. Plos One. (2017) 12.

67. Ochiai R, Saitou K, Suzukamo C, Osaki N, Asada T. Effect of chlorogenic acids on cognitive function in mild cognitive impairment: a randomized controlled crossover trial. J Alzheimers Dis. (2019) 72:1209-16. doi: 10.3233/Jad-190757

68. Pase MP, Scholey AB, Pipingas A, Kras M, Nolidin K, Gibbs A, et al. Cocoa polyphenols enhance positive mood states but not cognitive performance: a randomized, placebo-controlled trial. J Psychopharmacol. (2013) 27:4518. doi: $10.1177 / 0269881112473791$

69. Pipingas A, Silberstein RB, L Vitetta, C Rooy, Van, H.arris EV, Young JM, et al. Improved cognitive performance after dietary supplementation with a Pinus radiata bark extract formulation. Phytother Res. (2008) 22:5449. doi: $10.1002 /$ ptr. 2388

70. Rainey-Smith SR, Brown BM, Sohrabi HR, Shah T, Goozee KG, Gupta VB, et al. Curcumin and cognition: a randomised, placebo-controlled, doubleblind study of community-dwelling older adults. Br J Nutr. (2016) 115:210613. doi: $10.1017 / S 0007114516001203$

71. Ryan J, Croft K, Mori T, Wesnes K, Spong J, Downey L, et al. An examination of the effects of the antioxidant Pycnogenol (R) on cognitive performance, serum lipid profile, endocrinological and oxidative stress biomarkers in an elderly population. J Psychopharmacol. (2008) 22:553-62. doi: $10.1177 / 0269881108091584$

72. Santos RF, Galduroz JCF, Barbieri A, Castiglioni MLV, Ytaya LY, Bueno OFA. Cognitive performance, SPECT, and blood viscosity in elderly non-demented people using Ginkgo biloba. Pharmacopsychiatry. (2003) 36:127-33.

73. Schneider LS, DeKosky ST, Farlow MR, Tariot PN, Hoerr R, Kieser MA. Randomized, double-blind, placebo-controlled trial of two doses of Ginkgo biloba extract in dementia of the Alzheimer's type. Curr Alzheimer Res. (2005) 2:541-51. doi: 10.2174/156720505774932287

74. Schneider LS, Hernandez G, Zhao L, Franke AA, Chen YL, Pawluczyk S, et al. Safety and feasibility of estrogen receptor-beta targeted phytoSERM formulation for menopausal symptoms: phase $1 \mathrm{~b} / 2 \mathrm{a}$ randomized clinical trial. Menopause. (2019) 26:874-84. doi: 10.1097/GME.0000000000001325

75. Siddarth P, Li ZP, Miller KJ, Ercoli LM, Merril DA, Henning SM, et al. Randomized placebo-controlled study of the memory effects of pomegranate juice in middle-aged and older adults. Am J Clin Nutr. (2020) 111:170-7. doi: 10.1093/ajen/nqz241

76. Small BJ, Rawson KS, Martin C, Eisel SL, Sanberg CD, McEvoy CL, et al. Nutraceutical intervention improves older adults' cognitive functioning. Rejuvenation Res. (2014) 17:27-32. doi: 10.1089/rej.2013.1477

77. Snitz BE, O'Meara ES, Carlson MC, Arnold AM, Ives DG, Rapp SR, et al. Ginkgo biloba for preventing cognitive decline in older adults a randomized trial. J Am Med Assoc. (2009) 302:2663-70. doi: 10.1001/jama.2009. 1913

78. Solomon PR, Adams F, Silver A, Zimmer J, DeVeaux, R. Ginkgo for memory enhancement - a randomized controlled trial. JAMA. (2002) 288:835-40. doi: 10.1001/jama.288.7.835

79. Suominen MH, Laaksonen MML, Salmenius-Suominen $\mathrm{H}$, Kautiainen $\mathrm{H}$, Hongisto SM, Tuukkanen $\mathrm{K}$, et al. The short-term effect of dark chocolate flavanols on cognition in older adults: a randomized controlled trial (FlaSeCo). Exp Gerontol. (2020) 136:110933. doi: 10.1016/j.exger.2020.110933

80. Thaung Zaw JJ, Howe PR, Wong RH. Long-term effects of resveratrol on cognition, cerebrovascular function and cardio-metabolic markers in postmenopausal women: a 24-month randomised, double-blind, placebo-controlled, crossover study. Clin Nutr. (2021) 40:820-9. doi: 10.1016/j.clnu.2020.08.025
81. van Dongen MCJM, van Rossum E, Kessels AGH, Sielhorst HJG, Knipschild PG. The efficacy of ginkgo for elderly people with dementia and ageassociated memory impairment: new results of a randomized clinical trial. $J$ Am Geriatr Soc. (2000) 48:1183-94. doi: 10.1111/j.1532-5415.2000.tb02589.x

82. Whyte AR, Cheng N, Fromentin E, Williams CM. A randomized, doubleblinded, placebo-controlled study to compare the safety and efficacy of low dose enhanced wild blueberry powder and wild blueberry extract (think blue) in maintenance of episodic and working memory in older adults. Nutrients. (2018) 10:660. doi: 10.3390/nu10060660

83. Wightman EL, Jackson PA, Khan J, Forster J, Heiner F, Feistel B, et al. The acute and chronic cognitive and cerebral blood flow effects of a sideritis scardica (greek mountain tea) extract: a double blind, randomized, placebo controlled, parallel groups study in healthy humans. Nutrients. (2018) 10:955. doi: 10.3390/nu10080955

84. Witte AV, Kerti L, Margulies DS, Floel A. Effects of resveratrol on memory performance, hippocampal functional connectivity, and glucose metabolism in healthy older adults. J Neurosci. (2014) 34:786270. doi: 10.1523/JNEUROSCI.0385-14.2014

85. Wong RHX, Berry NM, Coates AM, Buckley JD, Bryan J, Kunz I, et al. Chronic resveratrol consumption improves brachial flowmediated dilatation in healthy obese adults. J Hypertens. (2013) 31:181927. doi: 10.1097/HJH.0b013e328362b9d6

86. Woo J, Lau E, Ho SC, Cheng F, Chan C, Chan ASY, et al. Comparison of Pueraria lobata with hormone replacement therapy in treating the adverse health consequences of menopause. Menopause. (2003) 10:352-61. doi: 10.1097/01.Gme.0000054764.94658.33

87. Yakoot M, Salem A, Helmy S. Effect of memo (R), a natural formula combination, on mini-mental state Examination scores in patients with mild cognitive impairment. Clin Interv Aging. (2013) 8:975-81. doi: $10.2147 /$ Cia.S44777

88. Yancheva S, Ihl R, Nikolova G, Panayotov P, Schlaefke S, Hoerr R, et al. Ginkgo biloba extract EGb 761, donepezil or both combined in the treatment of Alzheimer's disease with neuropsychiatric features: a randomised, doubleblind, exploratory trial. Aging Ment Health. (2009) 13:183-90.

89. You YX, Shahar S, Rajab NF, Haron H, Yahya HM, Mohamad M, et al. Effects of 12 weeks cosmos caudatus supplement among older adults with mild cognitive impairment: a randomized, double-blind and placebo-controlled trial. Nutrients. (2021) 13. doi: 10.3390/nu13020434

90. Zhang SJ, Xue ZY. Effect of Western medicine therapy assisted by Ginkgo biloba tablet on vascular cognitive impairment of none dementia. Asian Pac J Trop Med. (2012) 5:661-4. doi: 10.1016/S1995-7645(12)60135-7

91. JASP Team. JASP (Version 0.14.1) [Computer software] (Amsterdam). (2020).

92. Truzzi F, Tibaldi C, Zhang Y, Dinelli G, D' Amen E. An overview on dietary polyphenols and their biopharmaceutical classification system (BCS). Int $J$ Mol Sci. (2021) 22:5514. doi: 10.3390/ijms22115514

93. Cheng PF, Chen JJ, Zhou XY, Ren YF, Huang W, Zhou JJ, et al. Do soy isoflavones improve cognitive function in postmenopausal women? A meta-analysis. Menopause. (2015) 22:198-206. doi: 10.1097/GME.0000000000000290

94. File SE, Jarrett N, Fluck E, Duffy R, Casey K, Wiseman H. Eating soya improves human memory. Psychopharmacology. (2001) 157:4306. doi: $10.1007 / \mathrm{s} 002130100845$

95. Brickman AM, Khan UA, Provenzano FA, Yeung LK, Suzuki W, Schroeter $\mathrm{H}$, et al. Enhancing dentate gyrus function with dietary flavanols improves cognition in older adults. Nat Neurosci. (2014) 17:1798803. doi: $10.1038 / \mathrm{nn} .3850$

96. Ezzyat Y, Olson IR. The medial temporal lobe and visual working memory: comparisons across tasks, delays, and visual similarity. Cogn Affect Behav Neurosci. (2008) 8:32-40. doi: 10.3758/CABN.8.1.32

97. Braun M, Weinrich C, Finke C, Ostendorf F, Lehmann T-N, Ploner CJ. Lesions affecting the right hippocampal formation differentially impair short-term memory of spatial and nonspatial associations. Hippocampus. (2011) 21:309-18. doi: 10.1002/hipo.20752

98. Hannula DE, Tranel D, Cohen NJ. The long and the short of it: relational memory impairments in amnesia, even at short lags. J Neurosci. (2006) 26:8352-9. doi: 10.1523/JNEUROSCI.5222-05.2006

99. Kesse-Guyot E, Fezeu L, Andreeva VA, Touvier M, Scalbert A, Hercberg $\mathrm{S}$, et al. Total and specific polyphenol intakes in midlife are associated 
with cognitive function measured 13 years later. J Nutr. (2012) 142:7683. doi: $10.3945 /$ jn. 111.144428

100. Slopien R, Wender-Ozegowska E, Rogowicz-Frontczak A, Meczekalski B, Zozulinska-Ziolkiewicz D, Jaremek JD, et al. Menopause and diabetes: EMAS clinical guide. Maturitas. (2018) 117:6-10. doi: 10.1016/j.maturitas.2018.08.009

101. Liu K, Zhou R, Wang B, Mi M-T. Effect of resveratrol on glucose control and insulin sensitivity: a meta-analysis of 11 randomized controlled trials. Am J Clin Nutr. (2014) 99:1510-9. doi: 10.3945/ajcn.113. 082024

102. Davinelli S, Ali S, Solfrizzi V, Scapagnini G, Corbi G. Carotenoids and cognitive outcomes: a meta-analysis of randomized intervention trials. Antioxidants. (2021) 10:223. doi: 10.3390/antiox10020223

103. Medawar E, Huhn S, Villringer A, Veronica Witte A. The effects of plantbased diets on the body and the brain: a systematic review. Transl Psychiatry. (2019) 9:226. doi: 10.1038/s41398-019-0552-0

104. Carregosa D, Mota S, Ferreira S, Alves-Dias B, Loncarevic-Vasiljkovic N, Crespo CL, et al. Overview of beneficial effects of (poly)phenol metabolites in the context of neurodegenerative diseases on model organisms. Nutrients. (2021) 13:2940. doi: 10.3390/nu13092940

105. Hill BD, Elliott EM, Shelton JT, Pella RD, O'Jile JR, Gouvier WD. Can we improve the clinical assessment of working memory? An evaluation of the Wechsler Adult Intelligence Scale-Third Edition using a working memory criterion construct. J Clin Exp Neuropsychol. (2010) 32:315-23. doi: 10.1080/13803390903032529

106. Guyatt GH, Oxman AD, Vist GE, Kunz R, Falck-Ytter Y, AlonsoCoello P, et al. GRADE: an emerging consensus on rating quality of evidence and strength of recommendations. BMJ. (2008) 336:9246. doi: 10.1136/bmj.39489.470347.AD

107. Schäfer T, Schwarz MA. The meaningfulness of effect sizes in psychological research: differences between sub-disciplines and the impact of potential biases. Front Psychol. (2019) 10:813-6. doi: 10.3389/fpsyg.2019.00813

108. Stark SM, Stark CEL. Age-related deficits in the mnemonic similarity task for objects and scenes. Behav Brain Res. (2017) 333:109-17. doi: 10.1016/j.bbr.2017.06.049

Conflict of Interest: The authors declare that the research was conducted in the absence of any commercial or financial relationships that could be construed as a potential conflict of interest.

Publisher's Note: All claims expressed in this article are solely those of the authors and do not necessarily represent those of their affiliated organizations, or those of the publisher, the editors and the reviewers. Any product that may be evaluated in this article, or claim that may be made by its manufacturer, is not guaranteed or endorsed by the publisher.

Copyright (๑) 2022 de Vries, Medawar, Korosi and Witte. This is an open-access article distributed under the terms of the Creative Commons Attribution License (CC $B Y)$. The use, distribution or reproduction in other forums is permitted, provided the original author(s) and the copyright owner(s) are credited and that the original publication in this journal is cited, in accordance with accepted academic practice. No use, distribution or reproduction is permitted which does not comply with these terms. 